\title{
Dependence of shear wave seismoelectrics on soil textures: a numerical study in the vadose zone
}

\author{
F.I. Zyserman, ${ }^{1}$ L.B. Monachesi ${ }^{2}$ and L. Jouniaux ${ }^{3}$ \\ ${ }^{1}$ Facultad de Ciencias Astronómicas y Geofísicas, Universidad Nacional de La Plata y CONICET, Paseo del Bosque s/n, \\ B1900FWALa Plata,Argentina.E-mail: zyserman@fcaglp.unlp.edu.ar \\ ${ }^{2}$ Instituto de Investigación en Paleobiología y Geología, Universidad Nacional de Río Negro y CONICET, Av. Roca 1242, Gral. Roca, Río Negro, Argentina \\ ${ }^{3}$ Institut de Physique du Globe de Strasbourg, EOST, UdS-CNRS UMR 7516, Université de Strasbourg, 5 rue René Descartes, F-67084 Strasbourg, France
}

Accepted 2016 November 14. Received 2016 November 10; in original form 2016 May 12

\begin{abstract}
SUMMAR Y
In this work, we study seismoelectric conversions generated in the vadose zone, when this region is traversed by a pure $S H$ wave. We assume that the soil is a 1-D partially saturated lossy porous medium and we use the van Genuchten's constitutive model to describe the water saturation profile. Correspondingly, we extend Pride's formulation to deal with partially saturated media. In order to evaluate the influence of different soil textures we perform a numerical analysis considering, among other relevant properties, the electrokinetic coupling, coseismic responses and interface responses (IRs). We propose new analytical transfer functions for the electric and magnetic field as a function of the water saturation, modifying those of Bordes et al. and Garambois \& Dietrich, respectively. Further, we introduce two substantially different saturation-dependent functions into the electrokinetic (EK) coupling linking the poroelastic and the electromagnetic wave equations. The numerical results show that the electric field IRs markedly depend on the soil texture and the chosen EK coupling model, and are several orders of magnitude stronger than the electric field coseismic ones. We also found that the IRs of the water table for the silty and clayey soils are stronger than those for the sandy soils, assuming a non-monotonous saturation dependence of the EK coupling, which takes into account the charged air-water interface. These IRs have been interpreted as the result of the jump in the viscous electric current density at the water table. The amplitude of the IR is obtained using a plane $S H$ wave, neglecting both the spherical spreading and the restriction of its origin to the first Fresnel zone, effects that could lower the predicted values. However, we made an estimation of the expected electric field IR amplitudes detectable in the field by means of the analytical transfer functions, accounting for spherical spreading of the $\mathrm{SH}$ seismic waves. This prediction yields a value of $15 \mu \mathrm{V} \mathrm{m}^{-1}$, which is compatible with reported values.
\end{abstract}

Key words: Numerical approximations and analysis; Electrical properties; Hydrogeophysics; Permeability and porosity; Wave propagation.

\section{INTRODUCTION}

Characterization of the vadose zone, that is, the portion of the subsurface above the water table, is one of the most challenging tasks in hydrogeological and geophysical investigations. One of the main reasons is that flow and transport processes are very sensitive to water saturation, which shows remarkable variations in this zone (Alumbaugh et al. 2002; Turesson 2006). The most commonly used geophysical techniques for water detection in the vadose zone are electric resistivity imaging (ERI), ground-penetrating radar (GPR), nuclear magnetic resonance imaging (NMR) and Streaming Potential (SP) measurements. All these methods detect the presence of water in soils by detecting changes in ground physical prop- erties (e.g. electrical conductivity, dielectric permittivity and electrokinetic coupling; Jouniaux et al. 1999; Bano \& Girard 2001; Garambois et al. 2002; Pinettes et al. 2002; Alumbaugh et al. 2002; Darnet et al. 2003; Henry et al. 2003; Sailhac et al. 2004; Girard et al. 2005; Jouniaux et al. 2006; Turesson 2006; Allègre et al. 2012; Giampaolo et al. 2014; Ahmed et al. 2016). Most commonly, in order to investigate the soil and the vadose zone, electromagnetic tools, such as time domain reflectometry (TDR) and groundpenetrating radar have been employed, sometimes coupled with electrical resistivity surveys, because of the sensitivity of resistivity and dielectric permittivity to volumetric moisture content (Darnet \& Marquis 2004; Brovelli et al. 2005; Bavusi et al. 2006; Hayley et al. 2007). 
One of the most novel techniques that recently has spawned new interest in vadose zone studies is the seismoelectric method. This method aims at combining the sensitivity of the electric methods to the fluid content with the spatial resolution of the seismic method. Although the seismoelectric effect had been known for several decades (Ivanov 1939; Frenkel 1944; Martner \& Sparks 1959; Long \& Rivers 1975; Russell et al. 1997), it was after the work of Pride (1994), where a closed formulation for the electromagneticto-seismic and seismic-to-electromagnetic conversions were presented, that this field has achieved new interest. The seismoelectromagnetic method can be used for non-invasive subsurface exploration. It shows potential for detecting pore fluids such as water, oil, gas, and also helps to better characterize the subsurface in terms of porosity, permeability, and fractures, see for example, Hu et al. (2007), Thompson et al. (2007), Bordes et al. (2008), Valuri et al. (2012). It is well known that the induced signals are small and difficult to detect, and need filtering techniques (Butler \& Russell 2003; Warden et al. 2012). However, some reports display interesting results; for example, seismoelectric effects related to surface waves have been observed in field measurements by Strahser et al. (2011), while conducting a seismoelectric survey over an unconfined aquifer, Dupuis et al. (2007) reported that the most prominent signal was generated at the water table; later, Dupuis et al. (2009) confirmed the results of the previous work by measuring conversions of compressional seismic waves to electric signals in wells. However, they noted that partially cemented layers commonly found to straddle the water table in the field may have played a role in generating those interfacial responses. They also measured spatial and temporal polarity reversals of the interface responses (IRs), providing evidence in support of the conceptual model for seismoelectric conversions at interfaces. The reader can find in a recent book (Revil et al. 2015) or open-access reviews (Jouniaux \& Ishido 2012; Jouniaux \& Zyserman 2016) a detailed description on the manifold advances attained in this research area. In this paper we are interested in the vadose zone, so we describe below the studies concerning the seismoelectric effect in partially saturated media.

Water saturation $S_{w}$ affects both mechanical and electrical properties such as seismic velocity, seismic attenuation, electrical conductivity, and also the electrokinetic coupling $L$, so both the coseismic field and IR properties are expected to depend on the soil water content. Among previous works in partially saturated soils, Strahser et al. (2011) observed seismoelectric conversions in the field, as a function of water saturation, and proposed a transfer function between the electric field and the acceleration as a function of the water saturation. However the observations could not be performed in a large range of water content, leading to relatively scattered data. Zyserman et al. (2010) modelled electroseismic waves in sandstones saturated with two-phase oil/water or gas/water mixtures by resorting to an effective medium approach to determine the mixture's mechanical properties, that is, the effective fluid mechanical properties were established by performing a weighted average of those of the individual fluid phases. For the electrical conductivity, the dielectric permittivity and the electrokinetic coupling, the values taken by these parameters in the wetting phase, that is, water, were retained. Moreover Munch \& Zyserman (2016) numerically studied the effect of (D)NAPL contamination in the vadose zone, and interpreted the source for the interface response due to an impinging $\mathrm{SH}$-wave source not as the standard dipole-like one, but as a current sheet flowing along the interface.

Using the modelling approach introduced by Revil \& Linde (2006), which considers electrokinetic couplings as a function of the charge density, Jardani et al. (2010) numerically simulated the forward seismoelectric response over a stratified medium including a reservoir partially saturated with oil, introducing the partial saturation condition by dividing the excess of charges appearing in the right hand side of the mentioned system of equations by the water saturation $S_{w}$. Later, Revil \& Mahardika (2013) and Revil et al. (2014) used the same approach in a numerical study of water flooding monitoring experiments, to track the saturation front in time. Note, however, that these last works employ a relation between the excess charge density and permeability $Q_{V}=-\frac{C_{s 0} \sigma_{0}}{k_{0}}$, that, to our knowledge, has not been validated through independent measurements of permeability and charge density. Indeed this expression means that if the volumetric charge density is deduced from streaming potential coefficient measurements $C_{s 0}$ and the rock electrical conductivity $\sigma_{0}$, using this equation, it can lead to a dependence between $Q_{V}$ and permeability, if the variations of $C_{s 0} \sigma_{0}$ are not important compared to the variations of the permeability, as just shown by the formula. This equation has been used to calculate $Q_{V}$, using permeability values, to deduce that $Q_{V}$ is inversely related to the permeability. Therefore, using this equation to calculate $Q_{V}$ does not prove by itself the existence of a real link between both quantities. Moreover it is more appropriate to use the electric current density as a function of the pressure, rather than as a function of the velocity and $Q_{V}$ because the velocity is an extrinsic variable while pressure is a thermodynamic variable; any change in volume could change the results when not using the pressure. Finally, the CEC being not a well-defined property, limited by kinetics, arises much more uncertainties. Therefore this approach should not be used unless the relation between the excess charge density and the permeability is proved (Jouniaux \& Zyserman 2015, 2016).

Concerning wave propagation in partially saturated soils, Warden et al. (2013) extended Pride's theory by making the model parameters - the streaming potential coefficient (SPC), bulk electrical conductivity, fluid viscosity, etc.- saturation dependent; they compared the influence of these parameters using different saturation laws. Modifying the Generalized Reflection and Transmission Matrix method (GRTM) accordingly, they used this extension to analyse the response of a capillary fringe between a totally saturated layer and a partially saturated one. The authors concluded that an IR created by a saturation contrast between sand and sandstone may be easier to detect than a SE conversion occurring at the same boundary between sand and sandstone with the two units fully saturated. Moreover they proved that the conversions depend on the type of saturation transition existing between the partially saturated and fully saturated units. Recently Bordes et al. (2015), extended analytic seismoelectric transfer functions (Pride \& Haartsen 1996; Garambois \& Dietrich 2001) for unsaturated conditions, and performed laboratory experiments to analyse them, showing that the amplitude ratio of seismic to coseismic electric signals remain rather constant upon variations of $S_{w}$, which is consistent with the theoretical predictions.

In the present work we extend the seismoelectric formulation, and correspondingly the associated finite element numerical code used in Zyserman et al. (2015), to appropriately model the different partial saturation profiles present in the vadose zone when considering different soil textures, which we characterize following the USDA nomenclature. Assuming a given fixed water table depth we obtain the water saturation profile up from the water table for five different soil textures assuming static conditions. Using a shear wave source, we analyse the seismic response of the different soils, and the behaviour of relevant parameters, such as electrical conductivity and electrokinetic coupling; further, for the latter we 
consider two different saturation dependent behaviours published in the literature. Then, we compare our numerical predictions for the coseismic conversions with analytical transfer functions, and we analyse the behaviour of the interface response for the different soils presented.

\section{THEORETICAL BACKGROUND}

In this section we derive the saturation profile for the different soil textures, and afterwards show how all the model parameters are appropriately written for the partial saturation environment.

\subsection{Saturation profile}

Let us assume that the soil is a partially saturated 1-D porous medium where water flow obeys Richards' equation (Richards 1931)

$\frac{\partial}{\partial z}\left(K(h) \frac{\partial}{\partial z}(h+z)\right)-\phi \frac{\partial S_{w}(h)}{\partial t}=0$,

$z$ being the vertical coordinate defined positive downwards, $h$ the pressure head, $\phi$ the porosity (which is assumed to be independent of depth), $S_{w}$ the water saturation and $K$ the hydraulic conductivity. Note that both $S_{w}$ and $K$ are explicitly written as functions of the pressure head. The maximum value for $K$ occurs when $h \geq 0$. Under this condition the soil is completely saturated $\left(S_{w}=1\right)$, and the hydraulic conductivity can be related to the absolute permeability $k_{0}$ of the porous medium as follows:

$K(h)=K_{s}=\frac{\rho_{w} g k_{0}}{\eta_{w}}$,

where $\rho_{w}$ and $\eta_{w}$ are the density and viscosity of water respectively and $g=9.806 \mathrm{~m} \mathrm{~s}^{-2}$ is the gravity of Earth. Eq. (1) governs the water flow in a 1-D partially saturated porous medium under Richards' assumptions (Richards 1931). To solve this equation, initial and boundary conditions must be given, together with constitutive relations between $S_{w}, K$ and $h$. In this paper, the van Genuchten constitutive model is used to compute the water saturation as a function of pressure head as (van Genuchten 1980)

$S_{w}(h)= \begin{cases}{\left[1+(\alpha|h|)^{n}\right]^{\left(\frac{1}{n}-1\right)}} & h<0 \\ 1 & h \geq 0 .\end{cases}$

In eq. (3), $\alpha$ and $n$ are parameters of the model, and will take different values depending on the considered soil. In Table 1, we display the five soil textures considered in this work, classified according the USDA nomenclature, along with their corresponding associated parameters $\phi, \alpha, n$ and $K_{s}$, as loamy sand, sandy loam, silt loam, sandy clay, and silty clay loam.

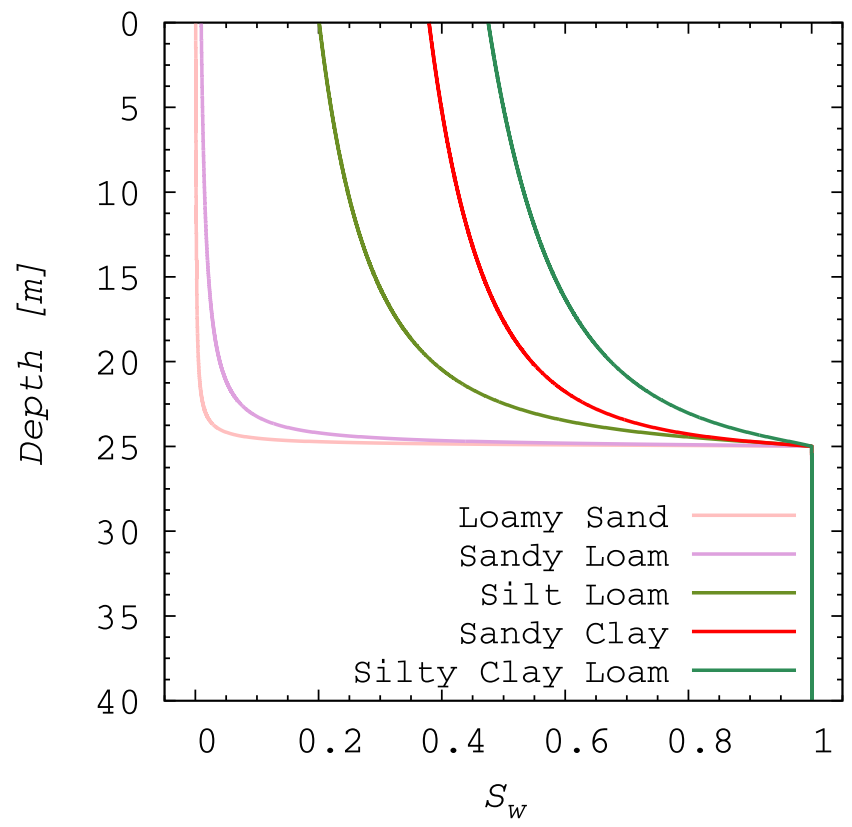

Figure 1. Saturation profile as calculated from eq. (5) for the five different soils. The water table is located at $z_{\mathrm{wt}}=25 \mathrm{~m}$.

Assuming a static situation, that is, the water in the vadose zone is not moving, $S_{w}$ cannot undergo variations in time, which reduces Richards' equation to

$\frac{\partial}{\partial z}\left(K(h) \frac{\partial}{\partial z}(h+z)\right)=0$.

Moreover, in this regime the flow predicted by Darcy's law $q=$ $-K(h) \frac{\partial}{\partial z}(h+z)$ must be zero. The solution for eq. (4) in this special case is given by $h+z=c$, where $c$ is a constant which may be determined upon the position of the zero-reference of the $z$-coordinate. Assuming that the origin of coordinates is located at the Earth's surface, as $h=0$ at the water table, then $c=z_{w t}$, which completely defines the pressure head profile for the static case. Therefore, the water saturation profile $S_{w}(z)$ can be obtained from the constitutive relation (3) and the $z$-dependence of the pressure head $h=z_{\mathrm{wt}}-z$

$$
S_{w}(z)= \begin{cases}{\left[1+\left(\alpha\left(z_{\mathrm{wt}}-z\right)\right)^{n}\right]^{\left(\frac{1}{n}-1\right)}} & z<z_{\mathrm{wt}} \\ 1 & z \geq z_{\mathrm{wt}} .\end{cases}
$$

Fig. 1 shows the water saturation profiles given by eq. (5) plotted for the above mentioned soil textures and assuming a water table depth $z_{\mathrm{wt}}=25 \mathrm{~m}$. The loamy sand and the sandy loam present a very sharp increase in the saturation profile in the immediate neighbourhood of the water table, while the more clayey textures, starting with a significant saturation at the surface, show a more gradual profile; however, the water saturation grows faster near $z_{\mathrm{wt}}$.

Table 1. Soils considered in this work, classified following the USDA nomenclature. Second to fourth columns display constituents fractions for the different textures. The corresponding hydraulic parameters for van Genuchten's saturation model $\left(\phi, \alpha, n\right.$ and $\left.K_{s}\right)$ are taken from Carsel \& Parrish (1998).

\begin{tabular}{lccccccc}
\hline Soil & $\gamma_{\text {sand }}$ & $\gamma_{\text {silt }}$ & $\gamma_{\text {clay }}$ & $\phi$ & $\alpha\left[\mathrm{cm}^{-1}\right]$ & $n$ & $K_{s}\left[\mathrm{~cm} \mathrm{~d}^{-1}\right]$ \\
\hline Loamy Sand & 0.75 & 0.20 & 0.05 & 0.41 & 0.124 & 2.28 & 350.2 \\
Sandy Loam & 0.55 & 0.30 & 0.15 & 0.41 & 0.075 & 1.89 & 106.1 \\
Silt Loam & 0.30 & 0.55 & 0.15 & 0.45 & 0.02 & 1.41 & 10.8 \\
Sandy Clay & 0.52 & 0.05 & 0.43 & 0.38 & 0.027 & 1.23 & 1.23 \\
Silty Clay Loam & 0.20 & 0.53 & 0.27 & 0.43 & 0.01 & 1.68 \\
\hline
\end{tabular}


Table 2. Values of model parameters used in this work. Those not shown in this table can be obtained from the present ones using the formulae described throughout the text.

\begin{tabular}{lr}
\hline \multicolumn{2}{c}{ Model parameters } \\
\hline$\hat{m}$ & 1.35 \\
$\hat{n}$ & 1.85 \\
$G_{\text {sand }}[\mathrm{Pa}]$ & 45 \\
$G_{\text {silt }}[\mathrm{GPa}]$ & 45 \\
$G_{\text {clay }}[\mathrm{GPa}]$ & 6.8 \\
$\rho_{s, \text { sand }}\left[\mathrm{Kg} \mathrm{m}{ }^{-3}\right]$ & 2600 \\
$\rho_{s, \text { silt }}\left[\mathrm{Kg} \mathrm{m}^{-3}\right]$ & 2600 \\
$\rho_{s, \text { clay }}\left[\mathrm{Kg} \mathrm{m}^{-3}\right]$ & 2580 \\
$D_{s, \text { sand }}[\mathrm{m}]$ & $8 \times 10^{-5}$ \\
$D_{s, \text { silt }}[\mathrm{m}]$ & $1 \times 10^{-5}$ \\
$D_{s, \text { clay }}[\mathrm{m}]$ & $5 \times 10^{-7}$ \\
$\eta_{w}[\mathrm{~Pa} \mathrm{~s}]$ & $1 \times 10^{-3}$ \\
$\rho_{w}\left[\mathrm{Kg} \mathrm{m}{ }^{-3}\right]$ & 1000 \\
$\eta_{a}[\mathrm{~Pa} \mathrm{~s}]$ & $1.81 \times 10^{-5}$ \\
$\rho_{a}\left[\mathrm{Kg} \mathrm{m}^{-3}\right]$ & 1.2 \\
$\epsilon_{w}\left[\mathrm{~F} \mathrm{~m}^{-1}\right]$ & $80 \epsilon_{0}$ \\
$C_{0}\left[\mathrm{~mol} \mathrm{~L}^{-1}\right]$ & $5 \times 10^{-3}$ \\
$T[\mathrm{~K}]$ & 298 \\
\hline
\end{tabular}

In the following section these curves will be used to compute the effective model parameters as functions of water saturation.

\subsection{Effective model parameters}

All classical model parameters are given in Tables 1 and 2; we explain here how all the valid ones in partial saturation conditions are derived from them. We consider three different materials building the solid matrix, namely sand, silt and clay, and we call $\gamma_{\text {sand }}, \gamma_{\text {silt }}$ and $\gamma_{\text {clay }}$ their respective volume fractions; $\gamma_{\text {sand }}+\gamma_{\text {silt }}+\gamma_{\text {clay }}=1$. The values of these fractions, for each soil type, are given in Table 1 . The mass density of the aggregate, $\rho_{s}$, is given by the volume weighted mean of the respective components mass densities,

$\rho_{s}=\gamma_{\text {sand }} \rho_{s, \text { sand }}+\gamma_{\text {silt }} \rho_{s, \text { silt }}+\gamma_{\text {clay }} \rho_{s, \text { clay }}$.

The absolute permeability value $k_{0}$ for each soil texture is computed from eq. (2) using material properties given in Tables 1 and 2. For the effective shear modulus of the aggregate $G_{s}$ we use the Reuss lower bound (Mavko et al. 2009), calculated in terms of the components volume fractions and shear moduli as:

$G_{s}=\left(\frac{\gamma_{\text {sand }}}{G_{s, \text { sand }}}+\frac{\gamma_{\text {silt }}}{G_{s, \text { silt }}}+\frac{\gamma_{\text {clay }}}{G_{s, \text { clay }}}\right)^{-1}$,

We use Walton model (Mavko et al. 2009) to estimate the solid matrix shear modulus $G$; this choice is appropriate when unconsolidated soils are considered (Pride 2005; Bordes et al. 2015; Dupuy et al. 2016). It reads

$G=\frac{1}{10}\left[\frac{3(1-\phi)^{2} \hat{C}^{2} P}{\pi B^{2}}\right]$, with $B=\frac{1}{4 \pi}\left(\frac{1}{G_{s}}+\frac{1}{G_{s}+\lambda_{c}}\right)$.

In this equation, $\hat{C}$ is the so called coordination number, and is related to the packing of the spheres building the solid aggregate, $P$ is the hydrostatic pressure and $\lambda_{c}$ is Lamé's coefficient of the effective grain material; in Walton model, it is calculated by taking the bulk modulus of the effective grain material equal to $\frac{5}{3} G_{S}$. In this work we consider $\hat{C}=9$ and $P=101325 \mathrm{~Pa}$.
In order to model the high attenuation levels of waves travelling through unconsolidated soils (Schön 1996), which are not explained - as we discuss in Section 5.1 - by Biot's intrinsic attenuation, we assume a linear viscoelastic behaviour for the bulk material using the correspondence principle as stated by Biot (Biot 1962; Carcione 2014), replacing the real shear modulus by a complex and frequency dependent viscoelastic shear modulus $G^{\star}$. The latter is obtained using Liu et al. (1976) linear viscoelastic model, as $G^{\star}(\omega)=G /(R(\omega)-i T(\omega))$; here $\omega$ is the angular frequency. The functions $R(\omega)$ and $T(\omega)$, associated with a continuous spectrum of relaxation times, characterize the viscoelastic behaviour and are given by (Santos et al. 2004)

$$
\begin{aligned}
& R(\omega)=1-\frac{1}{\pi \widehat{Q}} \ln \left(\frac{1+\omega^{2} T_{1}^{2}}{1+\omega^{2} T_{2}^{2}}\right), \\
& T(\omega)=\frac{2}{\pi \widehat{Q}} \tan ^{-1}\left(\frac{\omega\left(T_{1}-T_{2}\right)}{1+\omega^{2} T_{1} T_{2}}\right) .
\end{aligned}
$$

The model parameters $\widehat{Q}, T_{1}$ and $T_{2}$ are taken such that the soil quality factor $Q(\omega)$ is approximately equal to the constant $\widehat{Q}$ within the chosen frequency range. As we want to model a lossy medium, we consider $\widehat{Q}=30$, and as we work in the seismic frequency range, $T_{1}=\frac{1}{2 \pi} 10^{6} \mathrm{~s}$ and $T_{2}=\frac{1}{2 \pi} 10^{-7} \mathrm{~s}$ in our simulations. Although there are other models to account for the energy loss, for example, Chotiros \& Isakson (2004) and Gauzellino et al. (2014), we used Liu's model because it provides a constant quality factor over the frequency range considered, the modulus $G^{\star}$ is well behaved at $\omega \rightarrow 0$ and ensures causality, which is a reasonable behaviour for geophysical applications -unless otherwise expected in a particular model-, as suggested in Santos et al. (2005)

As our interest lies in both saturated and partially saturated porous media, it is necessary to introduce appropriate effective properties in order to use them within Pride's formulation for seismoelectrics. In order to do so, we follow here the same approach taken by Zyserman et al. (2012); Smeulders et al. (2014); Warden et al. (2013); Bordes et al. (2015); Zyserman et al. (2015). Let us start with the effective fluid mass density, for which we use (Bordes et al. 2015)

$\rho_{f}=\rho_{w} S_{w}+\rho_{a}\left(1-S_{w}\right)$,

where $S_{w}+S_{a}=1$ is assumed and subscript $a$ stands for air. The bulk density $\rho_{b}$ is then computed as

$\rho_{b}=\phi \rho_{f}+(1-\phi) \rho_{s}$.

The effective viscosity is computed in terms of the mixture components viscosities $\eta_{l}, l=w, a$ and water saturation $S_{w}$ using (Teja \& Rice 1981)

$\eta=\eta_{a}\left(\frac{\eta_{w}}{\eta_{a}}\right)^{S_{w}}$.

As usual, the shear modulus of the saturated rock matrix is assumed to be equal to $G$, the shear modulus of the dry matrix.

In order to characterize the electrical conductivity of the effective fluid saturated solid matrix we use the expression recently proposed by Warden et al. (2013), extending Pride's original formula (Pride 1994, eq. 242) to the realm of partially saturated media:

$\sigma\left(S_{w}, \omega\right)=\frac{S_{w}^{\hat{n}}}{F} \sigma_{w}+\frac{2}{F} \frac{C_{\mathrm{em}}+C_{\mathrm{os}}(\omega)}{\Lambda}$.

The first term in this equation is Archie's law for a partially saturated medium, where $F=\phi^{-\hat{m}}$ stands for the formation factor, $\hat{m}$ being the cementation exponent and $\hat{n}$ the Archie's saturation exponent. The electrical conductivity of water containing sodium chloride 
can be computed as $\sigma_{w}=\sum_{l=\mathrm{Na}^{+}, \mathrm{Cl}^{-}}\left(e z_{l}\right)^{2} b_{l} N_{l}$, where $e=1.6 \times$ $10^{-19} \mathrm{C}$ is the electron electric charge, and $z_{l}$ is the ions' valence, taken to be one for both species. The ions' mobility $b_{l}$ and concentration $N_{l}$ (depending on the salinity $C_{0}$ ) are calculated following Carcione et al. (2003). The second term accounts for the surface conductivity; in it, as Pride stated in his seminal work, the factor $C_{\text {em }}$ is the excess conductance associated with the electromigration of double layer ions; $C_{\mathrm{os}}(\omega)$ is the frequency-dependent electroosmotic conductance due to electrically induced streaming of the excess double-layer ions. Finally, $\Lambda$ is a parameter obtained from the condition $\left(\xi \alpha_{\infty} k_{0}\right) /\left(\phi \Lambda^{2}\right)=1$ (Pride 1994); the pore-geometry dependent factor $\xi$ lies in the range $4 \leq \xi \leq 8$ and in this work we consider $\xi=8$. We remark here that, as in Brovelli et al. (2005) and Warden et al. (2013), the surface conductivity is assumed to be independent of water saturation, because under realistic saturation ranges (residual water saturation $S_{w r} \geq 0.1$ ) the thickness of the wetting phase layer on the pore surface is always larger than the Debye length. This also means that all fluid related properties involved in the calculation of the surface conductivity and of the electrokinetic coupling -see below- are just those of water.

Again, following Warden et al. (2013), we propose for the effective fluid saturated media the following electrokinetic coupling:

$L_{0}\left(S_{w}\right)=-\frac{\phi}{\alpha_{\infty}} \frac{\epsilon_{w} \zeta}{\eta_{w}}\left(1-2 \frac{d}{\Lambda}\right) S_{w}^{\hat{n}} \mathcal{C}\left(S_{w}\right)$

In this equation $\alpha_{\infty}=\phi F$ is the tortuosity, $\zeta$ is the zeta potential, $d$ is the Debye length and $\mathcal{C}\left(S_{w}\right)$ is a function relating the streaming potential coefficient obtained under partial saturation conditions to the one corresponding to full saturation conditions, which is described in what follows. We mentioned in the Introduction that several authors have investigated the relation $\mathcal{C}\left(S_{w}\right)$ from both theoretical and experimental viewpoints. In the study of Strahser et al. (2011), different models for the SPC dependence on $S_{w}$ were considered, namely the ones by Perrier \& Morat (2000), Revil et al. (2007), Guichet et al. (2003) and Allègre et al. (2010). Characterizing this dependence by a power law has also been proposed to model the relative electrokinetic coefficient in an imbibition experiment (Saunders et al. 2008). Jackson (2010) used a capillary tube model making explicit the SPC dependence with water saturation, relative permeability and relative charge density. Further models derived from considerations on how the excess charge dragged by the water varies with water saturation may be mentioned, as those derived by Mboh et al. (2012) and Jougnot et al. (2015). Recently, Allègre et al. (2012) modelled both Richards' equation for hydrodynamics and Poisson's equation for electrical potential for unsaturated conditions, using a 1-D finite element method. They concluded, based on previous laboratory experiments (Allègre et al. 2010 2011), that the unsaturated electrokinetic coefficient should have a non-monotonous behaviour. Moreover, experimental results from periodic succession of drainage and imbibition cycles on sand suggested that the air-water interface that develops for unsaturated conditions gets polarized and therefore generates an electrical response (Allègre et al. 2014). Allègre et al. (2015) showed that the interface between water and air should also be taken into account, since this interface is negatively charged, as is the interface between the rock matrix and the water. Moreover, during a drainage the surface area of this interface does not decrease with decreasing water saturation, but first increases before decreasing, leading to a non-monotonic behaviour of the resulting SPC (Allègre et al. 2015).
In this work we use the following relations, which display two qualitatively distinct behaviours, namely a monotonic or nonmonotonic dependence with water saturation respectively:

$$
\mathcal{C}\left(S_{w}\right)=\left\{\begin{array}{cc}
\frac{1}{S_{w}^{\hat{n}}}\left(\frac{S_{w}-S_{\mathrm{wr}}}{1-S_{\mathrm{wr}}}\right)^{2} & \\
S_{\mathrm{wr}}=0.20 & (\text { Perrier \& Morat 2000) } \\
\left(\frac{S_{w}-S_{\mathrm{wr}}}{1-S_{\mathrm{wr}}}\right)\left[1+32\left[1-\left(\frac{S_{w}-S_{\mathrm{wr}}}{1-S_{\mathrm{wr}}}\right)\right]^{0.4}\right] & \quad \text { (Allégre et al. 2010). }
\end{array}\right.
$$

For water saturations below $S_{\mathrm{wr}}, \mathcal{C}\left(S_{w}\right)$ is set to zero. We compute the zeta potential as $\zeta=0.008+0.026 \log _{10}\left(C_{0}\right)$ (Pride $\&$ Morgan 1991), and consider a fixed salt concentration $C_{0}=5 \times$ $10^{-3} \mathrm{~mol} \mathrm{~L}^{-1}$ (Table 2), such that $\zeta=-52 \mathrm{mV}$ throughout the modelling study.

\section{1-D SHTE SEISMOELECTRIC EQUATIONS}

We model the electromagnetic response to seismic waves generated by a shear source travelling through a partially saturated medium using the equations derived by Pride (1994). In particular we assume a 1-D Earth, and as a seismic source upon it, a shearing force $F^{s}$ parallel to the $x$ axis acting on a whole horizontal infinite plane. Under these assumptions both the solid and fluid phases can only undergo displacements in the $x$ direction, with amplitudes depending only on depth $z$; no compressional waves can arise in this model. Note that under this geometrical assumptions, spherical spreading and Fresnel zone effects are not accounted for. Considering also that the electroosmotic feedback can be neglected (Hu \& Liu 2002; Haines $\&$ Pride 2006), the seismoelectic equations in the space-frequency domain, assuming an $e^{+i \omega t}$ time dependence reduce to

$$
\begin{aligned}
& \sigma E_{x}-\frac{\partial H_{y}}{\partial z}=i \omega \frac{\eta}{k_{0}} L_{0} u_{f, x}=j_{v}, \\
& \frac{\partial E_{x}}{\partial z}+i \omega \mu_{0} H_{y}=0, \\
& -\omega^{2} \rho_{b} u_{s, x}-\omega^{2} \rho_{f} u_{f, x}-\frac{\partial}{\partial z}\left(G^{\star} \frac{\partial u_{s, x}}{\partial z}\right)=F^{s}, \\
& -\omega^{2} \rho_{f} u_{s, x}-\omega^{2} g_{0} u_{f, x}+i \omega \frac{\eta}{k_{0}} u_{f, x}=0 .
\end{aligned}
$$

From now on, for the sake of simplicity, we denote $u_{s} \equiv u_{s, x}(z, \omega)$, $u_{f} \equiv u_{f, x}(z, \omega), E \equiv E_{x}(z, \omega)$ and $H \equiv H_{y}(z, \omega)$. In eq. (16) $\mu_{0}$ is the vacuum magnetic susceptibility (we assume no ferromagnetic minerals present) and in eq. (18) $g_{0}=\frac{1}{2} \alpha_{\infty} \rho_{f} / \phi$ is Biot's low frequency inertial coupling coefficient. The right hand side in eq. (15) is the streaming current density $j_{v}$, source of the electromagnetic signals, whereas $\sigma E$ is the conduction current. Note that if the EK coupling $L_{0}$ is zero there is no electromagnetic field induced by the seismic source $F^{s}$.

The use of the low frequency Biot's eqs (17) and (18) and static electrokinetic coupling $L_{0}$ is explained by the fact that for all soils, the Biot characteristic frequency,

$\omega_{c}=\frac{\eta \phi}{\rho_{f} k_{0} \alpha_{\infty}}$,

which depends on water saturation through the effective parameters involved, is well above the seismic frequency range for all scenarios 


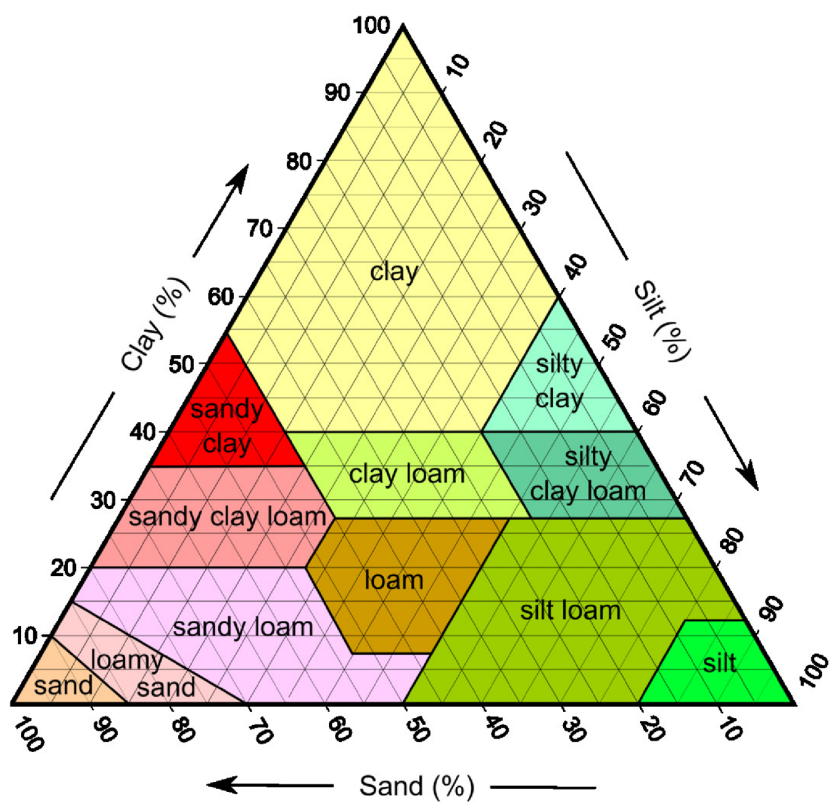

Figure 2. Soil texture triangle, taken from U.S. Soil Conservation Service (1987).

considered in this work. Following Santos et al. (2004), we write for $F^{s}$ in the right hand side of eq. (17)

$F^{s}(z, \omega)=\mathcal{F}(\omega) \frac{\partial \delta}{\partial z}\left(z-z_{f}\right)$.

$\mathcal{F}(\omega)$ is the Fourier transform of the seismic source time signature; by $z_{f}$ we denote the depth at which the source is located, and $\frac{\partial \delta}{\partial z}\left(z-z_{f}\right)$ represents the $z$-dependence of the force per unit volume produced by the shearing source.

We mention that the seismoelectric eqs (15)-(18) are equipped with absorbing boundary conditions in the computational domains, which minimize spurious reflections of impinging signals; see Zyserman et al. $(2012,2015)$ and references therein for details.

Finally, we note that as Pride (1994) warned, his set of seismoelectric equations are valid whenever the thin layer assumption is fulfilled; meaning that the thickness of the diffuse double layer, considered equal to the Debye length $d$, is much smaller than any geometrical length associated to the porous material. By using the possibility of varying the constituents' volume fractions within each soil texture allowed by the USDA classification (see Fig. 2), we have taken into account the aforementioned condition when choosing the volume fractions of constituents of the set of soil textures displayed in Table 1. To satisfy this condition, we required the effective particle size calculated as (Mavko et al. 2009)

$D_{s}=\left(\frac{\gamma_{\text {sand }}}{D_{s, \text { sand }}}+\frac{\gamma_{\text {silt }}}{D_{s, \text { silt }}}+\frac{\gamma_{\text {clay }}}{D_{s, \text { clay }}}\right)^{-1}$.

to be much bigger than the Debye length $d$; in this expression $D_{s, *}$ stands for the grain diameter of the different constituents.

\section{TRANSFER FUNCTIONS FOR E AND H FIELDS AS A FUNCTION OF WATER SATURATION}

We develop here the transfer functions for both the electric and the magnetic fields as functions of the water content. These analytical formulas will be compared to the numerical modelling in Section 7. Considering the low frequency regime, where displacement currents are negligible against conduction currents and seismic frequencies are much smaller than the Biot's characteristic frequency $\omega_{c}$, Garambois \& Dietrich (2001) derived theoretical transfer functions for compressional and shear waves, showing that they convey useful information about the properties of the porous media near receivers. These authors derived the relation between measured $\mathrm{SH}$ or $S V$ solid displacements and magnetic field in a fully saturated porous medium, given by (Garambois \& Dietrich 2001)

$|H| \simeq \omega \frac{\phi}{\alpha_{\infty}} \frac{\epsilon_{w} \rho_{w}|\zeta|}{\eta_{w}} \sqrt{\frac{G}{\rho_{b}}}\left|u_{s}\right|=\omega \mathcal{C}_{\mathrm{ek}}^{\mathrm{sat}} \sigma \rho_{w} \sqrt{\frac{G}{\rho_{b}}}\left|u_{s}\right|$,

where the SPC at full saturation is $\mathcal{C}_{\mathrm{ek}}^{\mathrm{sat}}=\epsilon_{w} \zeta /\left(\eta_{w} \sigma_{w}\right)$, usually measured in laboratory (Jouniaux et al. 1994; Maineult et al. 2006), and $\sigma=\sigma_{w} / F$, that is, in deriving the transfer function the surface conductivity is considered negligible compared to the bulk conductivity. Note that here we have written the fields in the frequency domain, instead of using the original time dependent expression. More recently, Bordes et al. (2015), based on the works of Pride \& Haartsen (1996) and Garambois \& Dietrich (2001), derived dynamic transfer functions relating the electric field amplitude with grain accelerations, for both compressional and shear waves. The low frequency transfer function between $\mathrm{SH}$ waves and electric field, also in fully saturated media, expressing the fields in the frequency domain reads (Bordes et al. 2015):

$|E| \simeq \omega \frac{\phi}{\alpha_{\infty}} \frac{\epsilon_{w} \rho_{w}|\zeta|}{\eta_{w}} \mu_{0} \frac{G}{\rho_{b}}\left|u_{s}\right|=\omega \mathcal{C}_{\mathrm{ek}}^{\text {sat }} \sigma \rho_{w} \mu_{0} \frac{G}{\rho_{b}}\left|u_{s}\right|$.

Further, the authors extended equation (23) for unsaturated conditions, following the approaches proposed by Barrière et al. (2012) and Warden et al. (2013) for the unsaturated SPC, that is, writing $\mathcal{C}_{\mathrm{ek}}\left(S_{w}\right)=\mathcal{C}_{\mathrm{ek}}^{\mathrm{sat}} \mathcal{C}\left(S_{w}\right)$, and using $\sigma\left(S_{w}\right)=\sigma S_{w}^{\hat{n}}$ :

$|E| \simeq \omega \mathcal{C}_{\mathrm{ek}}^{\mathrm{sat}} \sigma \rho_{f} \mu_{0} \frac{G}{\rho_{b}} \mathcal{C}\left(S_{w}\right) S_{w}^{\hat{n}}\left|u_{s}\right|$,

where of course the parameters $\rho_{f}$ and $\rho_{b}$ are computed under partially saturated conditions, as previously explained. We modify eq. (24) to take into account the viscoelastic behaviour of the rock matrix as:

$|E| \simeq \omega \mathcal{C}_{\mathrm{ek}}^{\mathrm{sat}} \sigma \rho_{f} \mu_{0} \operatorname{Re}\left(\frac{G^{\star}}{\rho_{b}}\right) \mathcal{C}\left(S_{w}\right) S_{w}^{\hat{n}}\left|u_{s}\right|$,

Analogously, we extend the transfer function for the magnetic field to the partially saturated viscoelastic realm, which now reads

$|H| \simeq \omega \mathcal{C}_{\mathrm{ek}}^{\mathrm{sat}} \sigma \rho_{f} \operatorname{Re}\left(\sqrt{\frac{G^{\star}}{\rho_{b}}}\right) \mathcal{C}\left(S_{w}\right) S_{w}^{\hat{n}}\left|u_{s}\right|$.

In Section 7 an analysis of the transfer functions given by eqs (25) and (26) is presented, along with the comparison between analytical and numerical results.

\section{SEISMIC RESPONSE ANALYSIS}

\subsection{Seismic velocity and attenuation}

The shear wave slowness is given by (Pride 2005)

$s(\omega)=\left(\frac{1}{G^{\star}}\left(\rho_{b}-\frac{\rho_{f}^{2}}{g_{0}-i \eta /\left(\omega k_{0}\right)}\right)\right)^{\frac{1}{2}}$,

and from it the shear wave phase velocity is calculated as

$v_{s}(\omega)=1 / \operatorname{Re}(s(\omega))$. 


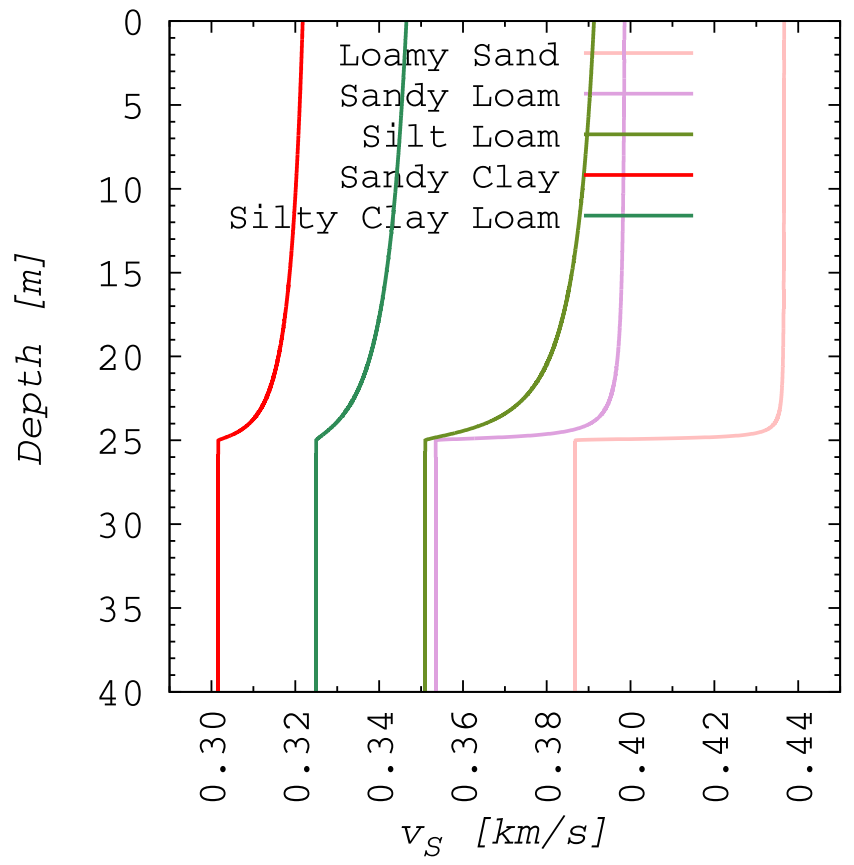

Figure 3. Shear wave phase velocity profile for the different soil textures, calculated at the source peak frequency $f_{c}$.

If we had not used the complex shear modulus $G^{\star}$ in eq. (27), the only contribution to the attenuation of the shear wave would have arisen from the relative fluid-solid motion induced by the acceleration of the rock matrix (Pride 2005), yielding high quality factor values $Q \approx 700$ for the soils we are studying. However, by introducing the viscoelastic behaviour of the rock matrix as explained in Section 2.2, we were able to set a value for the quality factor typical of unconsolidated soils, $Q=30$, for all considered soil textures.

In this work we take as the time signature of the seismic source a Ricker wavelet with peak frequency $f_{c}=120 \mathrm{~Hz}$; with this choice the signal is fairly sharp in time, and the different events are more easily resolved. The peak amplitude for the Ricker wavelet (located at $t=8 \times 10^{-3} \mathrm{~s}$ ) is set to $1 \mathrm{~N} \mathrm{~m}^{-3}$; note this is not a realistic value for a field source but it is appropriated for the qualitative analysis we perform in this work. An estimation of the absolute amplitude of the fields is undertaken in the Discussion. Fig. 3 display the phase velocity, for the different soil textures, as a function of depth, at the source peak frequency. The highest velocities are observed for

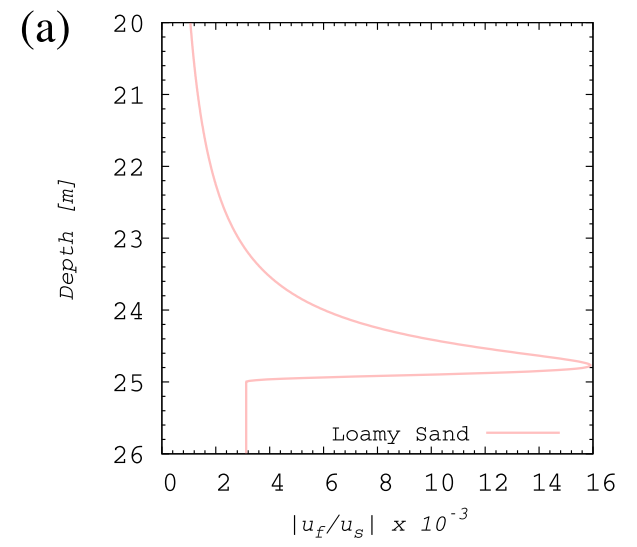

the sandy soils; while the clayey ones display lower speeds; for the silty clay loam $v_{s}$ is lower than $400 \mathrm{~m} \mathrm{~s}^{-1}$ at full saturation. Note that in all soils the increase of $S_{w}$ implies a fall in the wave propagation velocity, very strong near the water table for the loamy sand and sandy loam and less important and more gradual for the other textures.

The relative amplitudes between the solid displacement $u_{s}$ and the fluid displacement $u_{f}$ generated by the SH source play an important role in the amplitude of the induced electromagnetic response. From the shear wave dispersion relations for Biot's equations, this ratio can be calculated as (Pride 2005)

$\frac{u_{f}}{u_{s}}=i \omega \frac{\rho_{f} k_{0}}{\eta}$.

The behaviour of eq. (29) as a function of depth, computed at $\omega=2 \pi f_{c}$, for the loamy sand and the silty clay loam is displayed in Figs 4(a) and (b), respectively. The ratio $u_{f} / u_{s}$ is three orders of magnitude larger for the loamy sand compared to the silty clay loam, mainly because its higher permeability (two orders of magnitude). This result would imply that the seismoelectric signal is expected to be larger for the loamy sand than for the silty clay loam, at least for the coseismic signal. When considering the loamy sand, the displacements ratio increases with depth and as the wave approaches the water table it decays steeply to reach the saturated value. For the silty clay loam case the mentioned ratio decays monotonically with increasing water saturation, and as for the loamy sand, remains constant below the water table. This distinct behaviour is explained by the fact that for each one of the textures, $\rho_{f}$ and $\eta$ have different rates of increase with depth.

\subsection{Fluid and solid acceleration}

The numerical results of the seismic acceleration induced by the shear wave source, as a function of depth, are shown for the loamy sand (Figs 5a and b) and the silty clay loam (Figs 6a and b). The receivers are set from the surface downwards up to $50 \mathrm{~m}$ depth, separated one meter from each other. The solid acceleration amplitude decays as the seismic wave moves away from the source, for both soils, due to their relatively low quality factor. Almost no energy reflections occur at the water table, in agreement with Liu \& Greenhalgh (2014), who showed that a gas-water interface is almost transparent for small incident angles of an SV plane wave. Although these authors assume a sharp contrast in water saturation, their result can explain why in our case, the normal incident

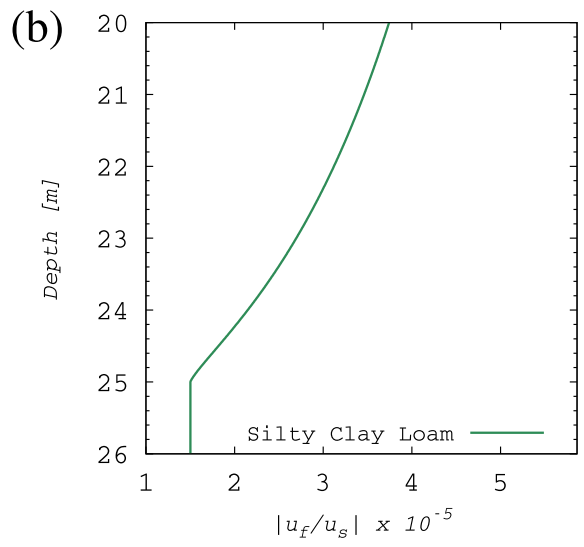

Figure 4. Relative fluid-solid motion profile calculated from eq. (29) for (a) loamy sand and (b) silty clay loam. 


\section{Loamy Sand}

(a)

Fluid Acceleration

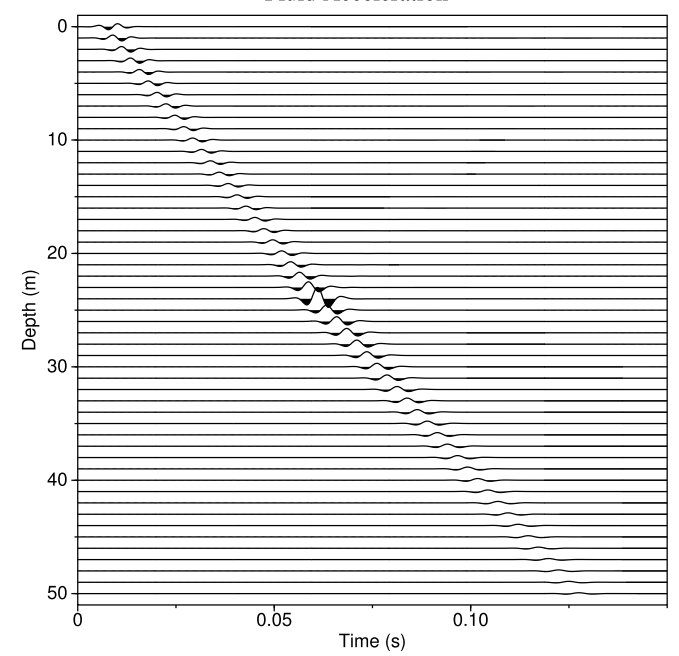

(c)

Electric Field

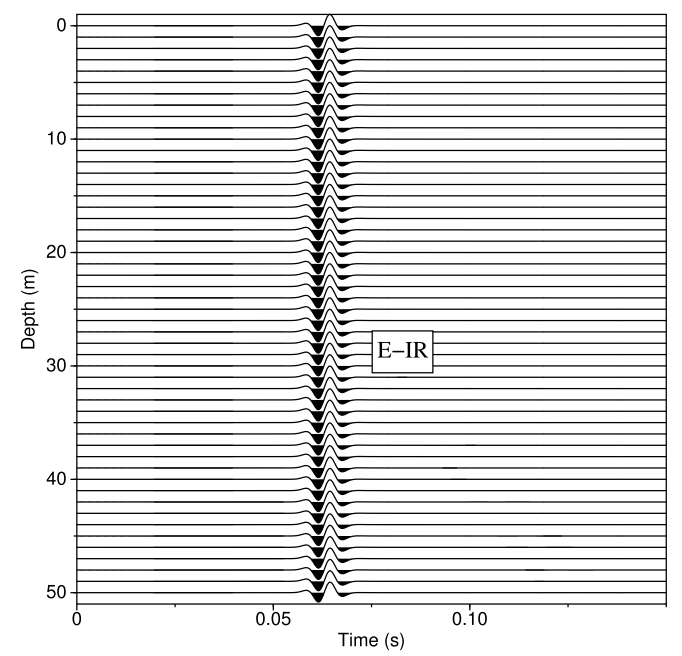

(b)

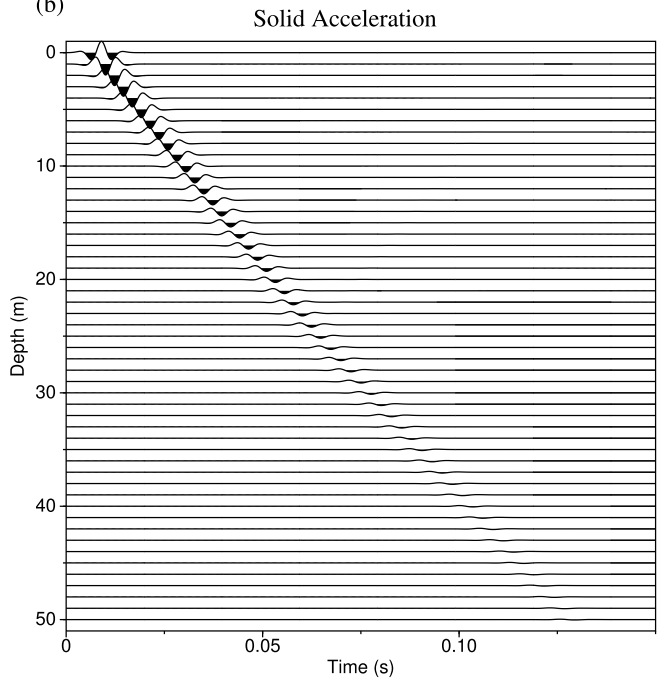

(d)

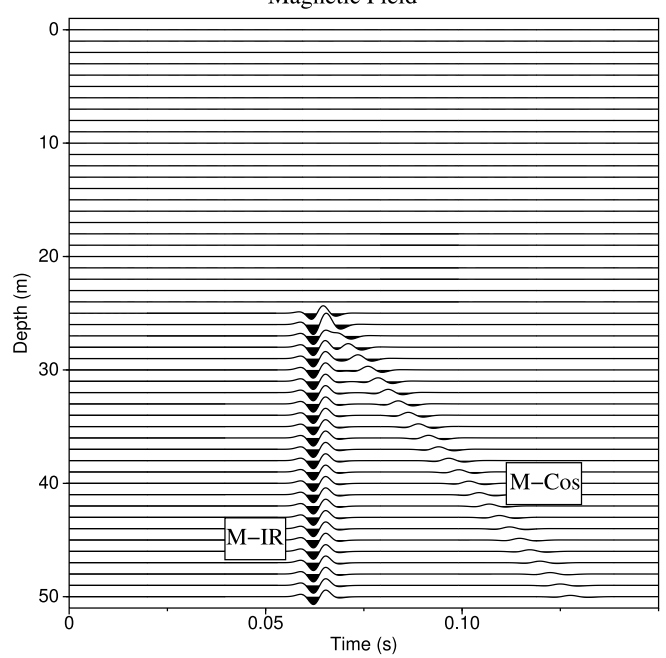

Figure 5. Loamy sand: numerical results for the fluid and solid acceleration, the electric and magnetic fields, induced by a shear wave source through the EK coupling, using the Allègre model for the SPC water saturation dependency.

shear wave does not produce any distinguishable reflection from the water table, as it is clear from Figs 5(a) \& (b), and 6(a) \& (b). The fluid acceleration also undergoes strong variations according to eq. (29); its behaviour is therefore one key point to explain the seismoelectromagnetic results. With respect to the loamy sand it can be noted how the amplitude of fluid acceleration reaches a maximum at $25 \mathrm{~m}$ and then decays slowly. On the other hand, in the silty clay loam case, the amplitude of the fluid acceleration monotonously decays with depth throughout the whole considered soil portion. This difference is not related to the different water saturation profiles for these soils (Fig. 1). These results are consistent with the markedly distinct behaviour of the relative amplitude between the solid and fluid displacement for these two different textures (Fig. 4), which depends on the effective density, permeability, and effective viscosity, as can be seen from eq. (29).

As it will be shown below, the fact that the soil texture influences the displacement of the fluid in the rock matrix, contributes to make the electromagnetic signal soil texture dependent.

\section{ELECTRICAL CONDUCTIVITY, VISCOSITY AND ELECTROKINETIC COUPLING ANALYSIS}

The electrical conductivity for all soils is constant below the water table, and the silty clay loam and silt loam are the most conductive ones (Fig. 7). The conductivity of the loamy sand and of the sandy loam shows a steep change near the water table, whereas the conductivity of the silty clay loam, sandy clay, and silt loam shows smoother variation and is higher at the surface. These results are clearly induced by the different saturation profiles (Fig. 1). The contribution of the surface conductivity to the total conductivity is not significant, mainly because of the relative high porosities characterizing the different textures, which makes the contribution of the bulk conductivity more important, even when the water salinity considered in this work is relatively low.

The effective viscosity, as expected, is also constant below the water table for all soils (Fig. 8). The behaviour of the effective viscosity with the depth, as in the previous case, follows the one 
Silty Clay Loam

(a)
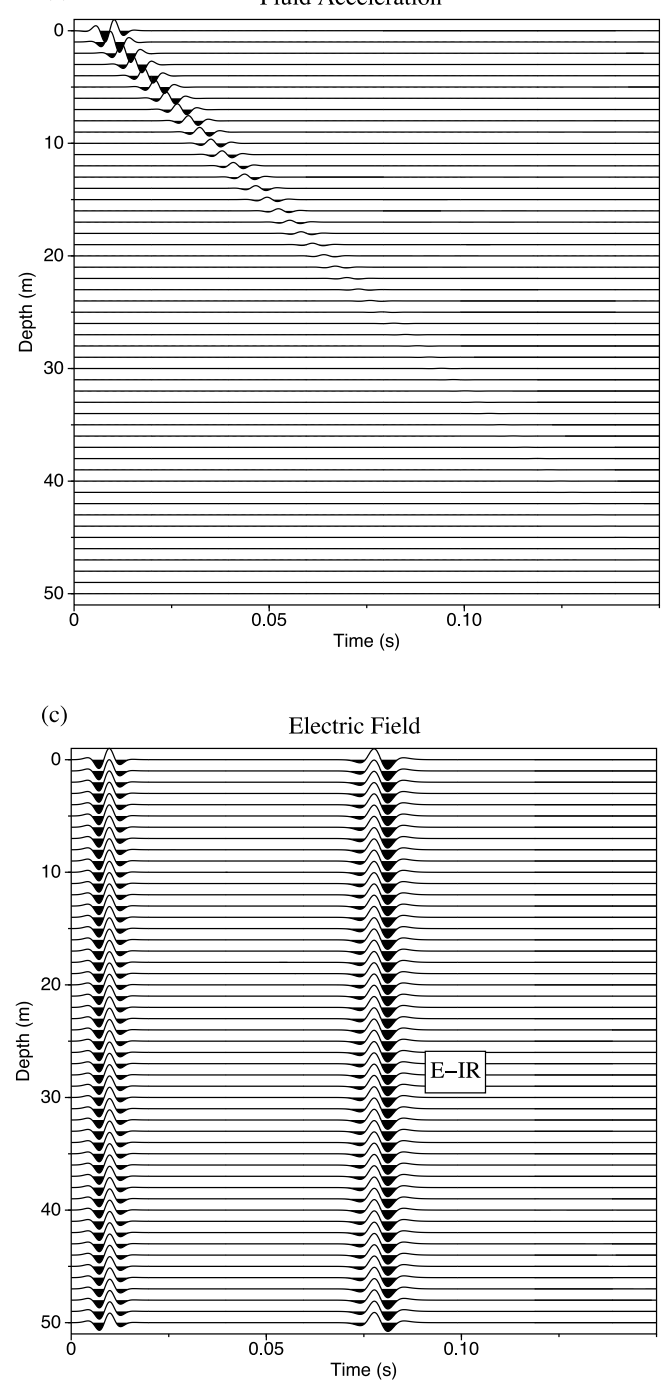

(b)
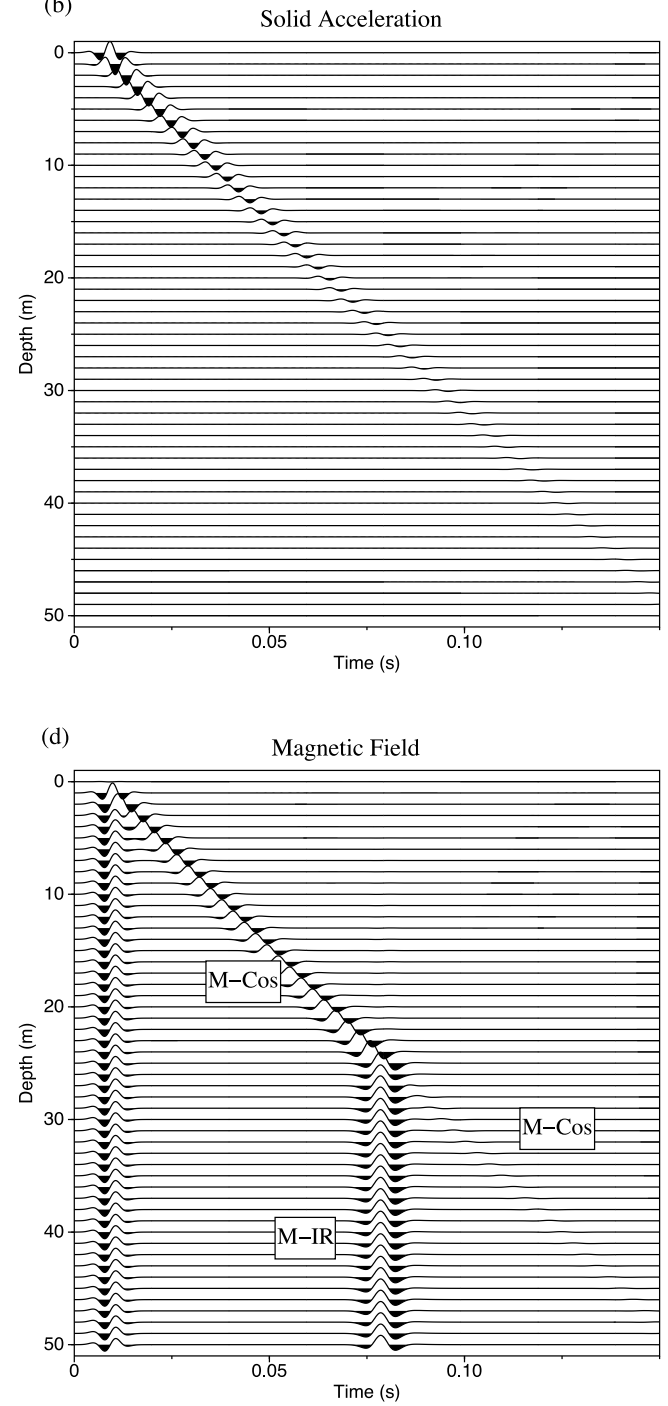

Figure 6. Silty clay loam: numerical results for the fluid and solid acceleration, the electric and magnetic fields, induced by a shear wave source through the EK coupling, using the Allègre model for the SPC water saturation dependency.

of the saturation profile; a steepy change near the water table is observed for the loamy sand and sandy loam of almost two orders of magnitude.

The electrokinetic coupling, which is the source of the seismoelectromagnetic conversions, is shown in Figs 9(a) and (b) for Perrier and Allègre models of the SPC water saturation dependence, respectively. The maximum value of the coupling is found for the Allègre model, leading to the conclusion that the seismoelectromagnetic conversions should be larger in this case, at least for the coseismic conversions. Using the Perrier model, the EK coupling increases with depth up to the water table, below which it is constant. The slope of this increase is much more important for the loamy sand and the sandy loam, inducing a larger contrast in $L$ at the water table, leading to the conclusion that the interfacial response should be larger for these soils when assuming the Perrier model. Using the Allègre model the EK coupling is increasing with the depth, and then suddenly decreases at the level of the water table. This behaviour is due to the non-monotonous behaviour of the SPC in the Allègre model. The jump at the water table may be more important for the silt loam, sandy loam, and silty clay loam, because the EK coupling above the water table keeps a large value.

\section{SEISMOELECTROMAGNETIC ANALYSIS: COSEISMIC AND IR RESPONSES}

\subsection{Seismoelectric analysis}

The results of the seismoelectric conversions induced by the shear wave source are shown for the loamy sand (Figs $5 \mathrm{c}$ and d) and for the silty clay loam (Figs $6 \mathrm{c}$ and d). The signals arriving at the same time for all receivers are interpreted as interfacial responses. The earliest one, observable for the silty clay loam for the electric and magnetic fields, is the interface response of the surface, which has no information of the subsurface, and has no interest in our study. This signal is not observable for the loamy sand case, because for this soil $L_{0}\left(S_{w}\right)=0$ from the surface down to several centimetres 


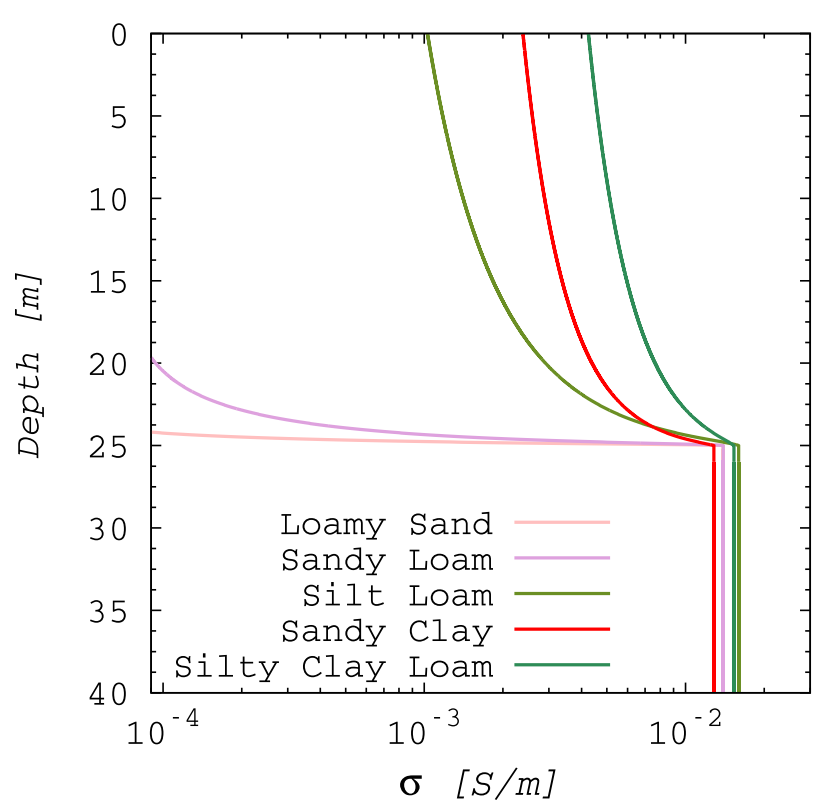

Figure 7. Effective electrical conductivity profile for the different soils.

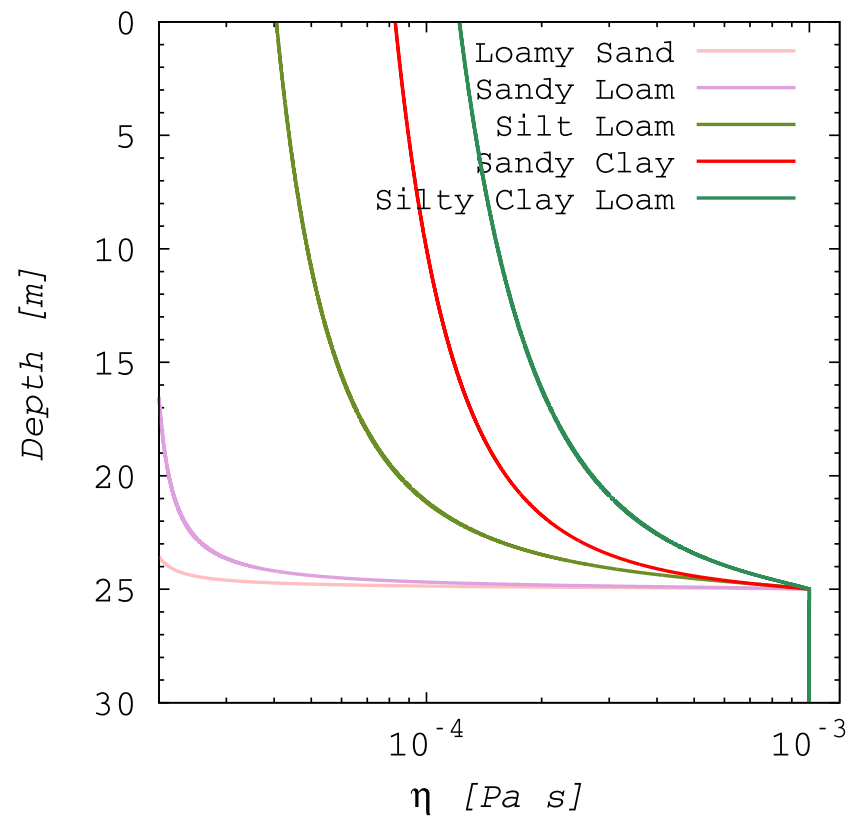

Figure 8. Effective viscosity profile for the different soils.

above the water table depth, which means that there is no seismoelectromagnetic coupling.

The arrival time for the IR of the loamy sand is about $0.06 \mathrm{~s}$, that is, the time at which the seismic wave arrives to the water table (Figs $5 \mathrm{a}$ and $\mathrm{b}$ ). The arrival time for the silty clay loam is about $0.075 \mathrm{~s}$, again consistent with the time at which the seismic wave impinges upon the water table (Figs 6a and b). In both soils the E-IR is the main electric signal, the amplitude of the coseismic signal being too low to be visible in the figure. In Fig. 10, the coseismic electric field signal arriving late in the record, is shown at an enlarged scale to show that the amplitude of the IR is three orders of magnitude larger than the coseismic amplitude. The three traces selected at depths of 45, 50, and $55 \mathrm{~m}$ show different arrival times of the coseismic responses, consistent with the velocity of the shear wave.
The key important result is that the seismoelectric conversions induced by a shear wave source lead to a large electric interfacial response compared to the coseismic signal, which is the opposite to what happens when using a $P$ wave source (Haines \& Pride 2006). Moreover, this E-IR can be measured on the surface and in a borehole, as can be seen in Figs 5(c) and (c).

\subsection{Seismomagnetic analysis}

The seismomagnetic results show both a coseismic signal (M-Cos) and an interfacial response (M-IR) (Figs 5d and 6d).

Coseismic magnetic field. The source for the coseismic signal is given, according to eq. (15), by the viscous current $j_{v}$, which drives an opposing conduction current $\sigma E$. The change in saturation with depth, affecting the different model parameters appearing in the right hand side of eq. (15), prevents the coseismic signal from strictly following the behaviour of the relative fluid displacement $u_{f}$, observable as accelerations in Figs 5(a) and 6(a). However, it can clearly be observed that at full saturation, the fluid acceleration and M-Cos signal depict the same behaviour for both textures: The coseismic signal amplitude decreases with depth due to the energy loss of the fluid displacement.

Magnetic IR. The amplitude of the mechanical source of the seismomagnetic conversion is not the main cause of the M-IR conversions; we associate the presence of these events to the jump occurring in the viscous current density in the neighbourhood of the water table. The jump observed in the amplitude of the M-IR is analogous to the one that occurs to the magnetic field generated by a current sheet; it is known that the difference between the magnetic field amplitude at both sides of the mentioned current sheet is proportional to the magnitude of the surface current density; moreover the amplitude of the magnetic field is higher on the more conductive side (although, of course, it decays also faster) (Munch \& Zyserman 2016, figs 1c and d). This interpretation explains also the fact that the E-IR is continuous across the water table; this behaviour is the one followed by the electric field at both sides of a current sheet. The magnetic IR is present both in the unsaturated and saturated regions, but it is stronger below the water table; therefore, this magnetic IR could be measured in a borehole below the water table, although it could be mixed with the coseismic part near $z_{\mathrm{wt}}$.

\subsection{Coseismic transfer functions}

The coseismic transfer functions of the electric and magnetic fields, calculated from eq. (25) and eq. (26), using the Perrier model, increase with increasing depth, up to a constant value below the water table (Figs 11a and c). The transition of these transfer functions to their value at full saturation is steeper for the loamy sand and the sandy loam. Using the Allègre model leads to an increase of the transfer function with increasing depth, with a sudden decrease at the water table level, reaching a constant value below $z_{\mathrm{wt}}$ (Figs 11 $\mathrm{b}$ and $\mathrm{d}$ ). The jump the transfer functions display at the water table for the loamy sand and the sandy loam cases is very abrupt, of several orders of magnitude. At the water table the values of the transfer function are one order of magnitude larger using the Allègre model, for both the electric and magnetic fields. Using this model, at full saturation the ratio between the electric field and the solid displacement is about $10^{-3} \mathrm{~V} \mathrm{~m}^{-2}$, as can be seen in Fig. 11(b). By dividing this value by the angular frequency $\omega$ squared, taken at $f_{c}=120 \mathrm{~Hz}$, yields about $2 \times 10^{-9} \mathrm{~V} \mathrm{~s}^{2} \mathrm{~m}^{-2}$; we can observe 
(a)
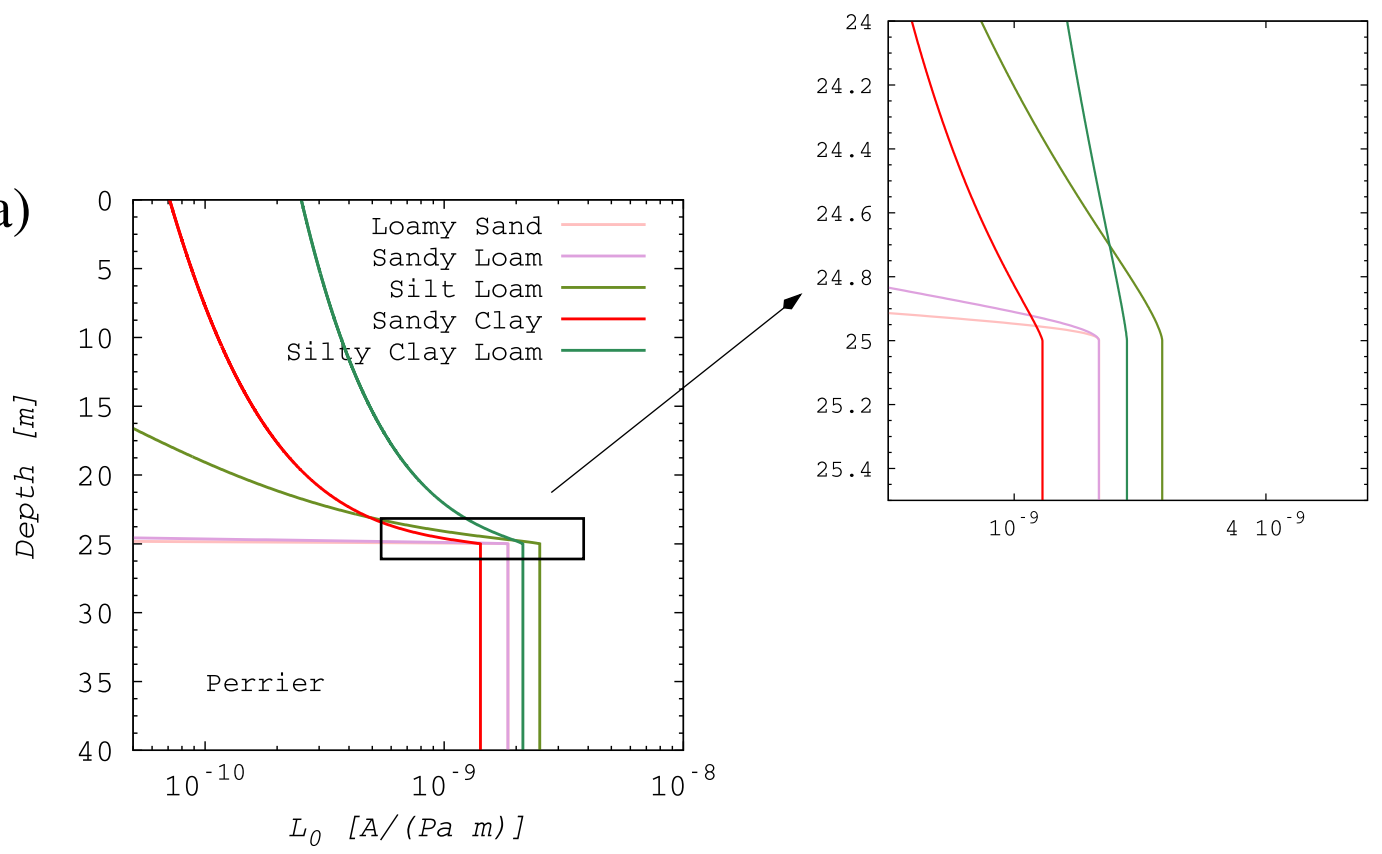

(b)

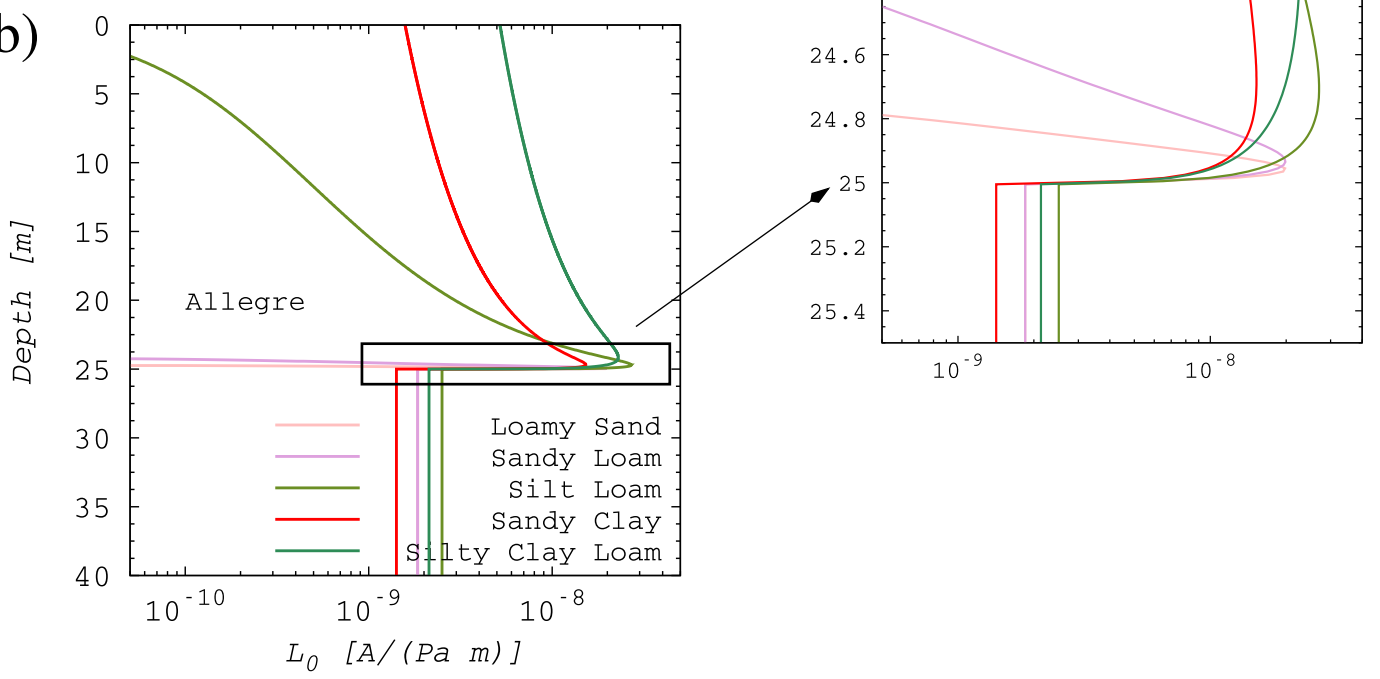

Figure 9. Electrokinetic coupling $L_{0}$, as a function of depth, for the different soils using (a) Perrier and (b) Allègre models.

that it is in very good agreement with the value of the $S$ waves transfer functions for the electric field over the acceleration at full saturation of about $10^{-10} \mathrm{~V} \mathrm{~s}^{2} \mathrm{~m}^{-2}$ reported by Bordes et al. (2015). The slight difference between these values comes from the different values employed for the shear moduli.

In Table 3, we display the coseismic ratio $|E| /|H|$, calculated from eqs (25) and (26) at the source peak frequency, namely $|E| /|H|=$ $\mu_{0} \operatorname{Re}\left(\sqrt{\frac{G^{\star}}{\rho_{b}}}\right)$, and from our numerical code, for the different soil textures at a position below the water table. In order to compute the numerical results, we proceeded as follows: Below the water table, as all results are equivalent, we chose a receiver at $50 \mathrm{~m}$ depth, so that we could easily select a time window which allows us to isolate both coseismic responses in the recorded traces. In this way, we avoid interferences with the interface response, which, being much stronger than the latter, would prevent us to compute the amplitude of the electric or magnetic field. We then compute the ratio $|E| /|H|$ taking for each one of the two traces the maximum positive peak of the coseismic signal. The analytical result shows that this ratio depends on the texture of the soil, but it does not depend on the saturation function $\mathcal{C}\left(S_{w}\right)$. That is, both Perrier and Allègre models should yield the same outcome, and this is what we observed from the numerical computations.

We consider now the dependence of the transfer functions with saturation $S_{w}$, again using the Allègre model. Fig. 12 shows that both transfer functions grow up to a water saturation of 90 per cent 


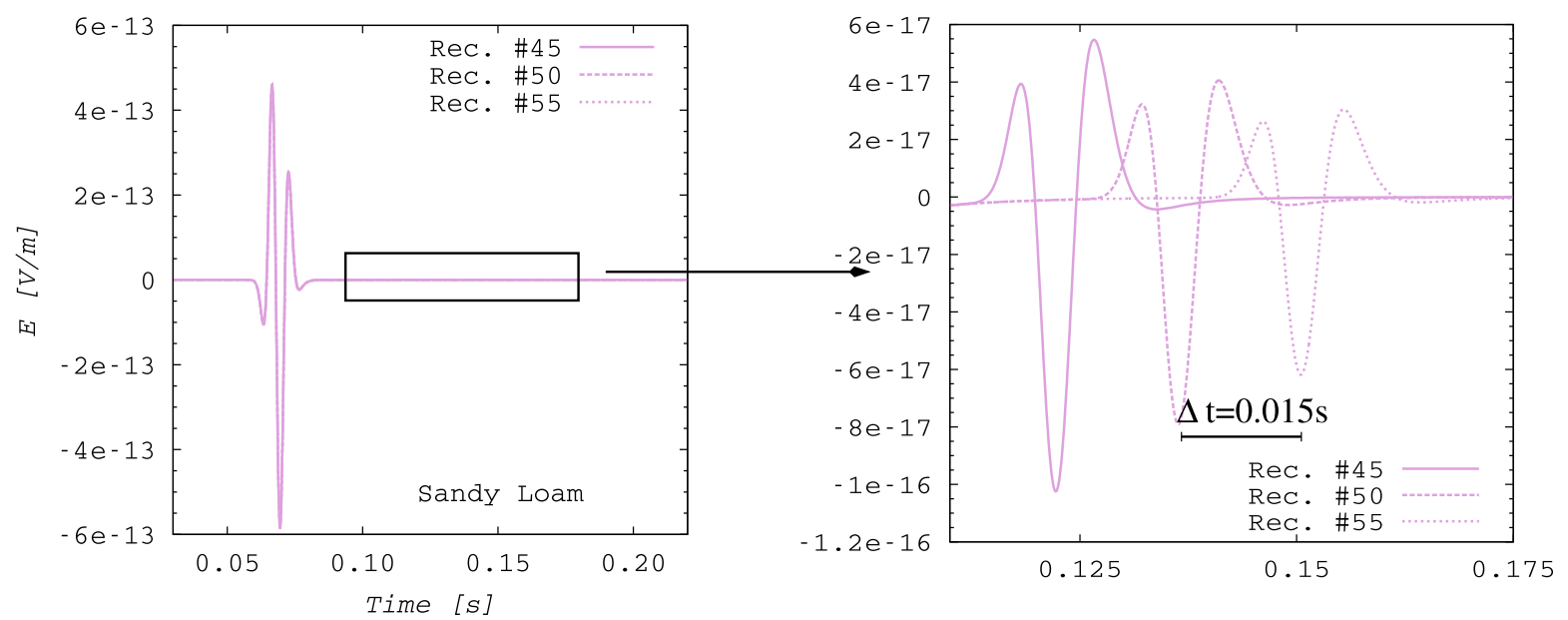

Figure 10. Electric field traces recorded at three different depths (receiver number denotes its depth in $\mathrm{m}$ ), for the sandy loam texture. As $v_{s}=353 \mathrm{~m} \mathrm{~s}{ }^{-1}$ at peak frequency below the water table and the distance between receivers $\# 45$ and $\# 50$ is $5 \mathrm{~m}$, the time shift between the signals depicted to the right is compatible with them being the E-Coseismic signal. Note that the amplitude of the latter is about four orders of magnitude smaller than the E-IR. The amplitudes are induced by the modelled source of $F=1 \mathrm{~N} \mathrm{~m}^{-3}$. The expected amplitude in the field is discussed in Section 8 .
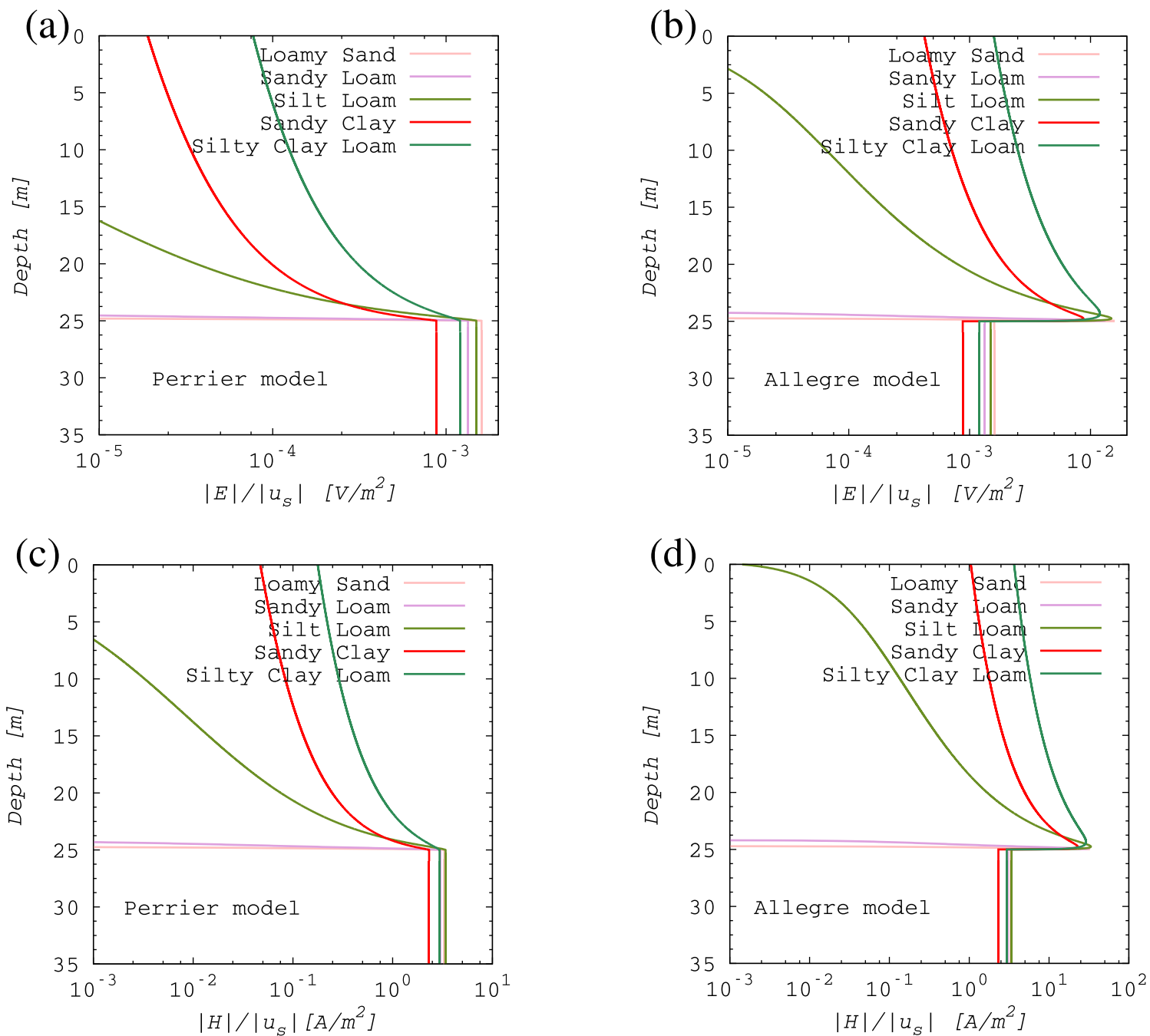

Figure 11. Electric field transfer function, as calculated by eq. (25), for (a) Perrier model and (b) Allègre model for the different soils here considered; and Magnetic field transfer function as calculated by eq. (26) for (c) Perrier model and (d) Allègre model. 
Table 3. Analytic and numeric ratios of the electric to magnetic coseismic fields, measured at $25 \mathrm{~m}$ below the water table.

\begin{tabular}{lcc}
\hline Saturated zone $|E| /|H|$ & Coseismic amplitudes [V/A] $\times 10^{-4}$ \\
\hline & Analytic & Numeric \\
\hline Loamy sand & 4.86 & 4.64 \\
Sandy loam & 4.44 & 4.51 \\
Silt loam & 4.41 & 4.03 \\
Sandy clay & 3.79 & 3.28 \\
Silty clay loam & 4.08 & 3.28 \\
\hline
\end{tabular}

and then decrease with increasing water saturation. The maximum enhancement is seen for sandy soils while the clayey soils depict the minimum one; these values differ in a factor of 2 to 2.5. Our results predict a magnetic transfer function at full saturation of about $3.5 \times 10^{-2} \mathrm{~A} \mathrm{~s} \mathrm{~m}^{-2}$ (Fig. 12b). The behaviour of the $S$-waves transfer functions as a function of the water saturation is qualitatively similar to the $P$ - waves electric transfer function proposed by Bordes et al. (2015).

\subsection{Interfacial response}

We detail in this section the observed differences in the IR among the considered soil textures and also study their dependence on to the viscous electric current density. In Fig. 13 we display traces for the electric and magnetic fields recorded at receivers located at $55 \mathrm{~m}$ depth, for all considered soil textures and using the two saturation models for $\mathcal{C}\left(S_{w}\right)$. The selected time window and depths ensure that the observed signals correspond solely to the interface responses. The amplitude of the E-IR and H-IR using the Allègre model are one order of magnitude larger than the one using the Perrier model for the fine to medium grained soils. When using the Perrier model the highest amplitudes of the IR occur for the coarse to medium grained soils (Figs 13a and b). The first two soils showed the largest jump in the EK coupling at the water table (Fig. 9a). Using the Allègre model the highest amplitudes of the IR are clearly for the silty clay loam and the silt loam, and are one order of magnitude larger than the ones for the loamy sand and the sandy loam (Figs 13c and d). This behaviour is difficult to understand in light of only the jump in the EK coupling.

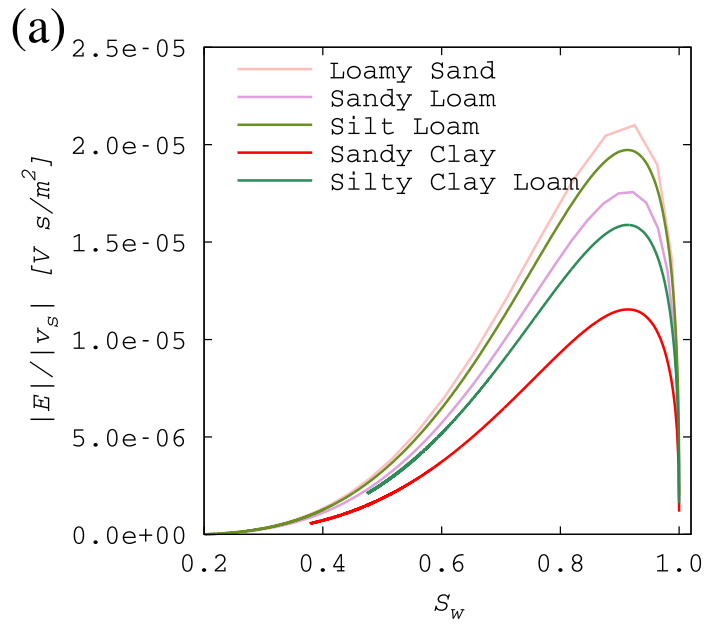

The viscous current density, depending on the EK coupling, the fluid displacement, the effective viscosity, and the permeability, as shown in the right hand side of eq. (15), presents different behaviours depending on the soil texture and on the model used for SPC water saturation dependence, as can be seen in Fig. 14. In the Allègre model case the current density is one order of magnitude larger than the one in Perrier model. Using the Perrier model it is not straightforward to explain the amplitude of the IR of the different soils on behalf of the current density. However, using the Allègre model it is clear that the small jump in the current density at the water table corresponds to a small IR for the loamy sand and the sandy loam. It is also clear that a large jump in the electric current density at the water table corresponds to a large IR amplitude for the silty clay loam and the silt loam, for both the electric and magnetic IR.

\section{DISCUSSION}

In this section we discuss the effect of different parameters on the seismoelectromagnetics conversions of the different soils, first on coseismic conversions, then on interfacial responses. Then we discuss the possibility of using $S$-wave source and the expected amplitudes of the interfacial responses.

The mechanical source is the first origin of these conversions, inducing a relative motion between the matrix and the fluid, leading to an electrokinetic coupling. The larger the relative motion, the larger the electromagnetic conversions are expected to be. However, these conversions are related to other parameters that can have a large influence. The amplitude of the EK coupling (eq. 13) directly controls the amplitude of the coseismic seismoelectric responses (eq. 15). The EK coupling, being equal to the SPC multiplied by the rock conductivity, also controls the transfer functions (eqs 25 and 26).

The IR is sensitive to the contrast in physical properties at the interface. It is expected that a large jump of the EK coupling or the electric current density induces a large IR amplitude. The EK coupling depends on the zeta potential, the fluid viscosity, the formation factor, the fluid saturation, and the electric current density depends further on the fluid displacement, the effective viscosity, and the permeability. All these parameters can therefore influence the seismoelectromagnetic conversions, and we discuss below why

(b)

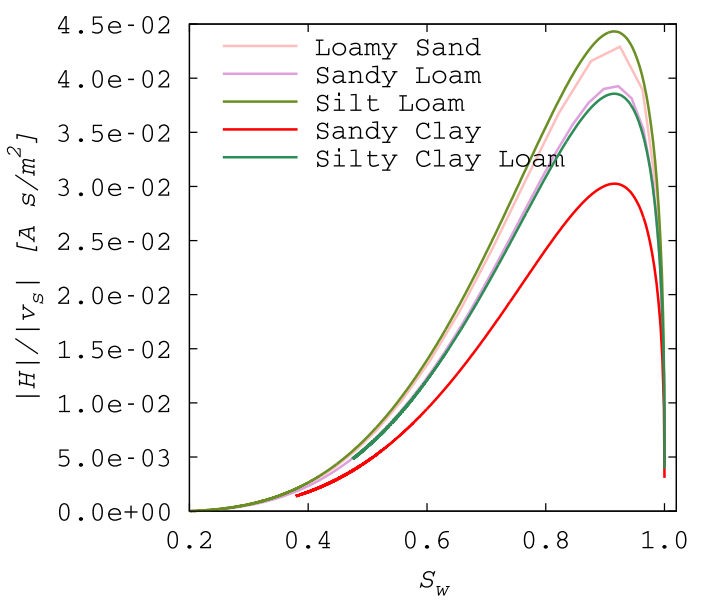

Figure 12. Transfer function dependence with saturation, considering the Allègre model; (a) Electric case and (b) Magnetic case. The considered frequency is the source peak one, $f_{c}=120 \mathrm{~Hz}$. 
(a)

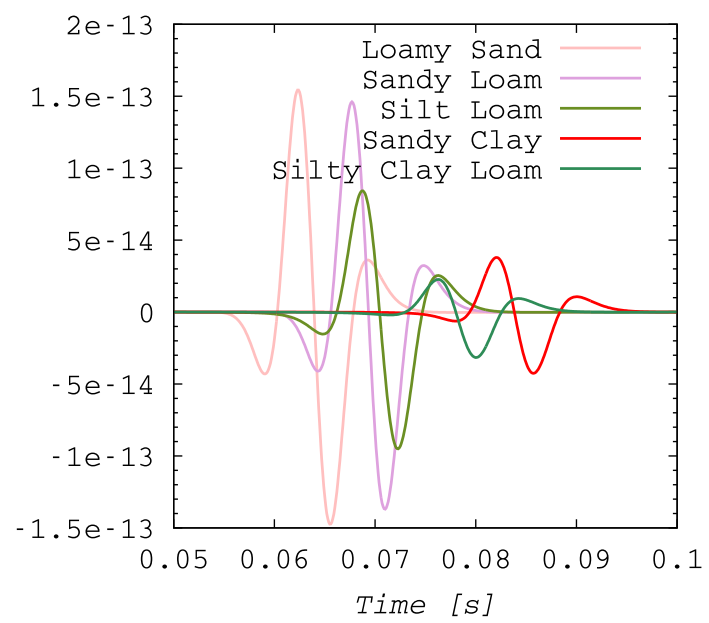

(c)

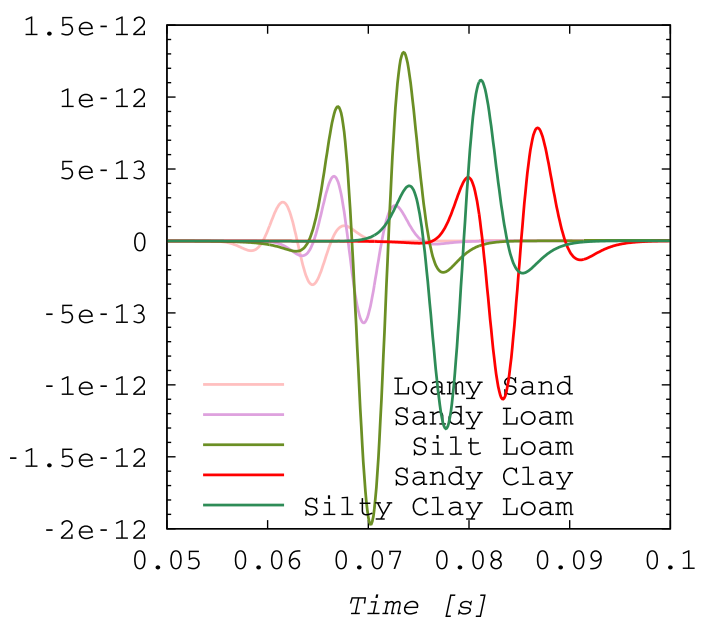

(b)

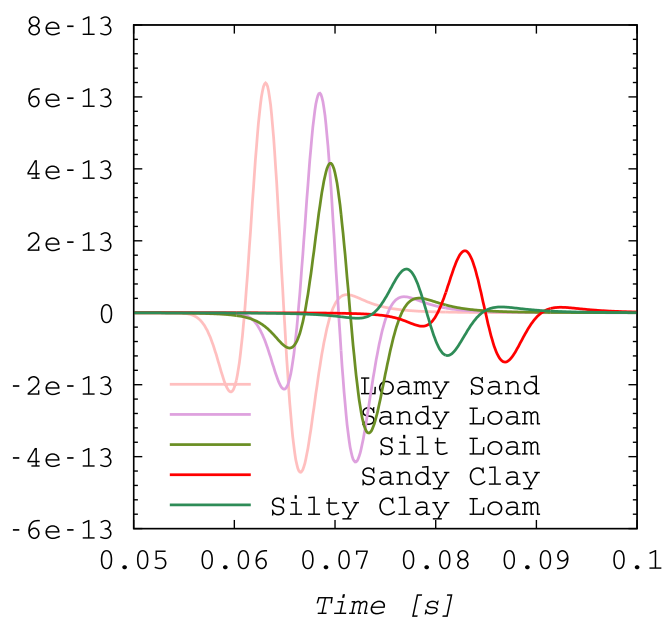

(d)

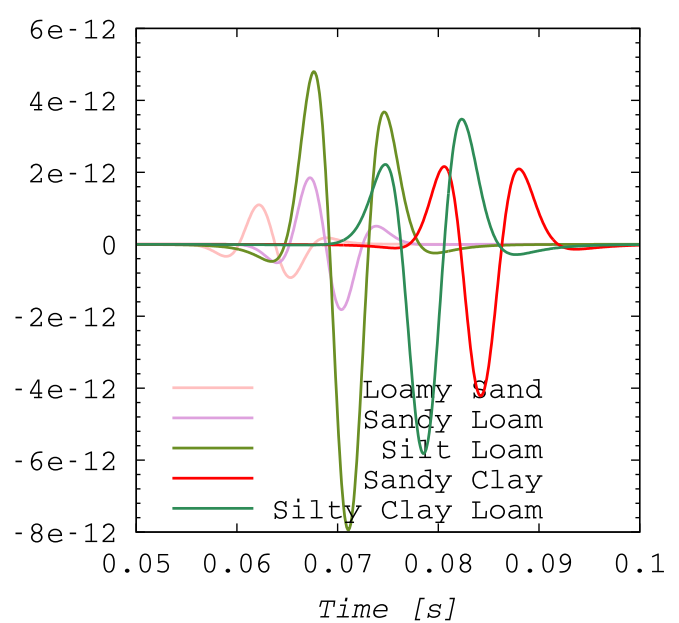

Figure 13. Electric and magnetic IR for the different soil textures and the two saturation models employed in this work. Traces are registered at $55 \mathrm{~m}$ depth; this value is chosen to avoid displaying the coseismic signals.

(a)

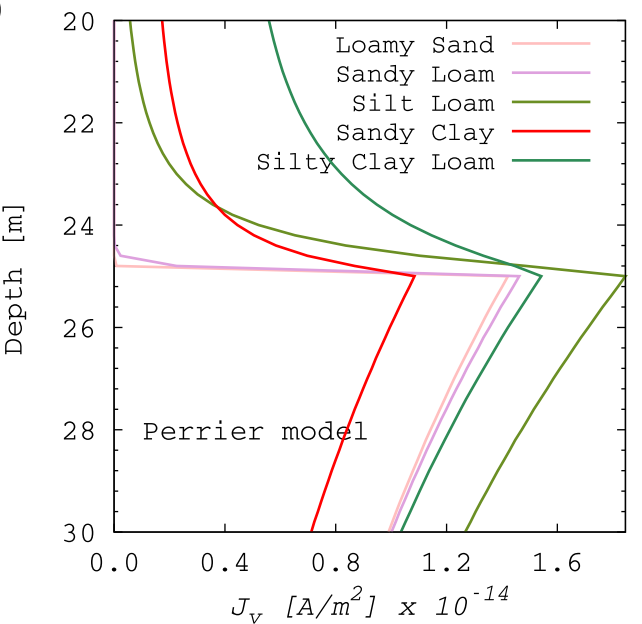

(b)

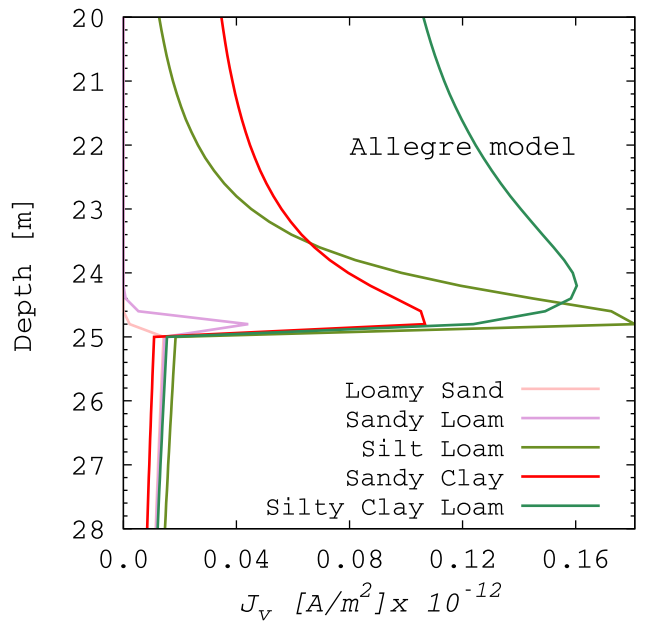

Figure 14. Current density depth dependence for the different soil textures and the two saturation models employed in this work, (a) Perrier model, (b) Allègre model. 
the seismoelectric conversions can be different for different soils in the vadose zone.

The seismic source of these conversions is an $\mathrm{SH}$ wave source. The shear wave source induces a matrix motion, leading to the dragging of the fluid because of the viscosity. There is no charge separation as appears using a $P$ wave source (Pride $\&$ Haartsen 1996; Haines \& Pride 2006). The induced electrical current forms horizontal current sheets, which are different mainly because they depend on the saturation profile. These current sheets lead to the coseismic magnetic field whose time variation generates the E-Cos signal, through Faraday's law eq. (16); recall that the amplitude of the ECos is very small, as we have shown in this work and other authors have previously observed (Haines \& Pride 2006). The jump near the water table of these currents leads to the magnetic IR, and the time variation of the M-IR, again through Faraday's law, creates the E-IR. Of course, being the conductivity of the medium not negligible, the induced conduction currents $\sigma E$ do also contribute to the fields M-IR and E-IR.

The maximum E-IR and H-IR are observed assuming the Allègre model, being two orders of magnitude larger than the ones assuming the Perrier model for at least the fine grained soil textures, namely the silty clay loam, the sandy clay and the silt loam. However, these amplitudes cannot be explained solely by the jump in the EK coupling, but by the jump in the amplitude of the viscous current density which is one order of magnitude larger for the Allègre model.

It is noteworthy that, when using the Allègre model, silty and clayey soils showed the highest interfacial responses both for the electric field and magnetic fields. This result is not intuitive since a larger relative motion between matrix and water is expected in sandy soils rather than in silty and clayey ones, which show a lower permeability. However, we showed that this mechanical source was complicated by the EK coupling and finally by the electric current density.

It is worth to note that Bordes et al. (2015) stated that the $S$-waves being converted very poorly into electric field it is hopeless to work with an $S$-wave seismoelectric field. These authors wrote about the coseismic magnitude of the E field induced by an $S$-wave, which we agree is very small compared to the amplitude of the E field induced by a $P$-wave, as they stated. However we showed that the E-IR response is much higher than the E-Cos response when using a $S$-wave source, which is the reverse when we use a $P$-wave source. Therefore it may be interesting to test $S$-wave source in the field.

We propose to use a pure SH seismic source that could achieve a better resolution than the one obtained through the usual P-driven experiments because of shorter wavelengths. Although a pure shear wave source is difficult to achieve, surface vibrators for SH-source do exist, which can emit $\mathrm{SH}$-waves as well as $\mathrm{SV}$-waves, being designed for a peak force of approximately $0.5 \mathrm{kN}$ and a frequency range well beyond the usually used in exploration geophysics, enabling resolutions, for shallow targets, up to ten times better than those attainable with $P$-wave sources (Krawczyk et al. 2012). Such a source has been successfully used to map sinkholes in urban areas (Krawczyk et al. 2013). Recently other $S$-wave source, also for shallow investigations, has been presented by Crane et al. (2013). Furthermore, there is presently interest in the seismics community in $S$-wave exploration because of its application in unconventional reservoirs. An application in heavy oil production management through $S$-wave data monitoring of stress effects in the reservoir has been reported (Bale et al. 2013), which could contribute to facilitate further progress in field measurements in seismoelectrics using shear wave sources.
The electric noise of the source can be a problem. When using a hammer, the triggering using the electric signal of the output of an accelerometer mounted on the hammer can generate electromagnetic noise. The manual triggering does not induce this noise. However several techniques of filtering can be used to manage the automatic triggering (Butler 1996; Butler et al. 2007). The hydraulically source may generate electromagnetic noise also. However it has been shown that using an hydraulic vibrator the interfacial response due to a water table at $14 \mathrm{~m}$ depth could be detected without any stacking at offsets up to $120 \mathrm{~m}$. When the data were stacked the IR of the base of the aquifer at depth between 40-60 m was shown on a profile up to $800 \mathrm{~m}$ (Dean et al. 2012; Valuri et al. 2012). Therefore it is worth to test a source with a higher strength than a hammer, even if this source induces some electromagnetic noise.

What would be the expected amplitudes of the interfacial response? The peak force of $P$-wave seismic source is usually such that the acceleration is about 3 or 4 times $g$ (the acceleration of gravity) (i.e. between 29.4 and $39.2 \mathrm{~m} \mathrm{~s}^{-2}$ ) (Lerwill 1981), and it can decrease up to $0.3 \mathrm{~g}$ at a few metres from the source. A shear wave generator such as ELVIS 5.0 (Krawczyk et al. 2013) with a peak force about $500 \mathrm{~N}$, would produce an acceleration of about $1.4 \mathrm{~g}$ at the source location, assuming a source weight of $35 \mathrm{Kg}$. This value could decrease to $0.05 \mathrm{~g}$ at $25 \mathrm{~m}$ from the source owing to spherical spreading. A vibrator truck $S$-wave source can produce an acceleration of about $0.4 \mathrm{~g}$ at a distance of $5 \mathrm{~m}$ (Bordes 2005). Therefore, accounting only for the amplitude loss by spherical spreading, it is reasonable to assume that the acceleration at $25 \mathrm{~m}$ by a shear source generator will be not higher than $0.08 \mathrm{~g}$. Then, the expected velocity at $120 \mathrm{~Hz}$ will not be higher than $0.001 \mathrm{~ms}^{-1}$. From Fig. 12 a maximum value for the ratio $|E| /\left|v_{s}\right|$ considering all textures is approximately $1.5 \times 10^{-5} \mathrm{Vs} \mathrm{m}^{-2}$. Then, the amplitude for the coseismic electric field is:

$|E|=0.015 \mu \mathrm{V} \mathrm{m}^{-1}$.

Finally, given that the amplitude of the interface response that we observed is about 3 orders of magnitude bigger than the coseismic signal, then the amplitude for the IR is:

$|E|=15 \mu \mathrm{V} \mathrm{m}^{-1}$,

Note that Dupuis et al. (2007) measured an E-IR from a water table at $14 \mathrm{~m}$ depth of about $0.6 \mu \mathrm{V} \mathrm{m}^{-1}$, using a weight drop source generating both $P$ and $S$ waves. Warden et al. (2013) used a point source $S$-wave to model an E-IR from a water table covered by a capillary fringe at $30 \mathrm{~m}$ depth of about $0.5 \mu \mathrm{V} \mathrm{m}{ }^{-1}$. It must be noted that our model uses a plane $S H$ wave undergoing no spherical spreading. Therefore the E-IR is coming from the whole interface rather than just the Fresnel zone, that may overestimate the calculated amplitude. Taking account for the spherical spreading, the amplitude predictions for the E-IR are compatible with the mentioned field measurements.

Concerning the H-Cos amplitude, we can say that our numerical results practically coincide with the theoretical estimates from Bordes et al. (2015) and Garambois \& Dietrich (2001), as we show in Table 3, where values of the ratio between electric and magnetic transfer functions at full saturation, that is, eq. (25) and eq. (26) with $S_{w}=1$, are given.

Assuming the same velocity estimation of $10^{-3} \mathrm{~m} \mathrm{~s}^{-1}$ produced by the $\mathrm{SH}$ wave at the water table, an estimation for the H-Cos amplitude can be made multiplying $3.5 \times 10^{-2} \mathrm{~A} \mathrm{~s} \mathrm{~m}^{-2}$ (transfer function value taken from Fig. 12b) by the estimated velocity, which gives $3.5 \times 10^{-5} \mathrm{~A} \mathrm{~m}^{-1}$. The corresponding value for the amplitude of the H-IR is in the same order magnitude as the H-Cos signal; 
this can be seen from the traces of the magnetic field recorded below the water table (a plot of these traces was not included in the paper; however, this can be appreciated in Figs $5 \mathrm{~d}$ and $6 \mathrm{~d}$ ). The estimated values for H-IR are higher than the ones reported by Garambois et al. (2002); however this comparison is difficult to be done, because their estimates correspond to compressional seismic sources, that is, H-IR generated by 'electric dipoles', which not necessarily would create magnetic fields with the similar amplitudes as the ones here considered, risen by jumps in the viscous current density.

However, Bordes et al. (2008) measured a magnetic field B of about $0.035 \mathrm{nT}$ for a $1 \mathrm{~m} \mathrm{~s}^{-2}$ seismic source acceleration, corresponding to $\mathrm{H}=\frac{1}{\mu_{0}} \mathrm{~B}=2.9 \times 10^{-5} \mathrm{~A} \mathrm{~m}^{-1}$, which is almost coincident with our estimation.

\section{CONCLUSIONS}

One key important result is that the seismoelectric conversions induced by a shear wave source lead to an electric interfacial response of about three orders of magnitude larger than the coseismic signal amplitude. Moreover, this E-IR can be measured both on the surface and in a borehole. The seismomagnetic IR is better detected below the water table, especially for sandy soils showing a sharp saturation profile. As the Allègre model takes into account the air-water interface in its non-monotonous behaviour with the water saturation, one main result is that interface responses of the water table for silty and clayey soils are larger than the ones for sandy soils, both for the electric and magnetic fields.

We propose to use a pure SH seismic source that could achieve a better resolution than the one obtained through the usual P-driven experiments because of shorter wavelengths.

The expected amplitude of the electric IR is $|E|=15 \mu \mathrm{Vm}^{-1}$, which is compatible with reported values. Detection of depth of the water table may be performed by surface measurements of the electric IR and surface measurements of the coseismic magnetic field especially for silty and clayey soils. Methods such as georadar detect easier the water table in almost all cases with the exception of clayey soils; then, combining both methods could improve the deduction of the water content within the soils. Further developments could study interfaces between different soils, taking into account the saturation profile.

\section{ACKNOWLEDGEMENTS}

We thank both anonymous reviewers, whose detailed comments largely helped to improve our work. FZ acknowledges support from CONICET through grant PIP 112-201501-00192. We thank INSUCNRS and the University of Strasbourg for their support.

\section{REFERENCES}

Ahmed, O.A., Derriche, Z., Kameche, M., Bahmani, A., Souli, H., Dubujet, P. \& Fleureau, J., 2016. Electro-remediation of lead contaminated kaolinite: an electro-kinetic treatment, Chem. Eng. Process. Process Intensification, 100, 37-48.

Allègre, V., Jouniaux, L., Lehmann, F. \& Sailhac, P., 2010. Streaming Potential dependence on water-content in fontainebleau sand, Geophys. J. Int., 182, 1248-1266.

Allègre, V., Jouniaux, L., Lehmann, F. \& Sailhac, P., 2011. Reply to the comment by A. Revil and N. Linde on: 'Streaming potential dependence on water-content in fontainebleau sand', Geophys. J. Int., 186, 115-117.
Allègre, V., Lehmann, F., Ackerer, P., Jouniaux, L. \& Sailhac, P., 2012. Modelling the streaming potential dependence on water content during drainage: 1. A 1D modelling of SP using finite element method, Geophys. J. Int., 189, 285-295.

Allègre, V., Maineult, A., Lehmann, F., Lopes, F. \& Zamora, M., 2014. Selfpotential response to drainage-imbibition cycles, Geophys. J. Int., 197, 1410-1424.

Allègre, V., Jouniaux, L., Lehmann, F., Sailhac, P. \& Toussaint, R., 2015. Influence of water pressure dynamics and fluid flow on the streamingpotential response for unsaturated conditions, Geophys. Prospect., 63, 694-712.

Alumbaugh, D., Chang, P.Y., Paprocki, L., Brainard, J., Glass, R. \& Rautman, C., 2002. Estimating moisture contents in the vadose zone using crossborehole ground penetrating radar: a study of accuracy and repeatability, Water Resour. Res., 38, 45-1-45-12.

Bale, R., Marchand, T., Wilkinson, K., Wikel, K. \& Kendall, R., 2013. The signature of shear-wave splitting: theory and observations on heavy oil data, Leading Edge, 32(1), 6434-6443.

Bano, M. \& Girard, J., 2001. Radar reflections and water content estimation of aeolian sand dune, Geophys. Res. Lett., 28(16), 32073210 .

Barrière, J., Bordes, C., Brito, D., Sénéchal, P. \& Perroud, H., 2012. Laboratory monitoring of $\mathrm{p}$ waves in partially saturated sand, Geophys. J. Int., 191(3), 1152-1170.

Bavusi, M., Rizzo, E. \& Lapenna, V., 2006. Electromagnetic methods to characterize the Savoia di Lucania waste dump (Southern Italy), Environ. Geol., 51, 301-308.

Biot, M.A., 1962. Mechanics of deformation and acoustic propagation in porous media, J. Appl. Phys., 34(1), 36-40.

Bordes, C., 2005. Etude expérimentale des phénomènes transitoires sismoélectromagnétiques: Mise en oeuvre au Laboratoire Souterrain à Bas Bruit de Rustrel, PhD thesis, Université Joseph Fourier, Grenoble 1.

Bordes, C., Jouniaux, L., Garambois, S., Dietrich, M., Pozzi, J.-P. \& Gaffet, S., 2008. Evidence of the theoretically predicted seismo-magnetic conversion, Geophys. J. Int., 174, 489-504.

Bordes, C., Sénéchal, P., Barrière, J., Brito, D., Normandin, E. \& Jougnot, D., 2015. Impact of water saturation on seismoelectric transfer functions: a laboratory study of coseismic phenomenon, Geophys. J. Int., 200, 13171335.

Brovelli, A., Cassiani, G., Dalla, E., Bergamini, F., Pitea, D. \& Binley, A.M., 2005. Electrical properties of partially saturated sandstones: novel computational approach with hydrogeophysical applications, Water Resour. Res., 41, W08411, doi:10.1029/2004WR003628.

Butler, K., 1996. Seismoelectrics effects of electrokinetic origin, PhD thesis, Univ. B.C. Vancouver, Canada.

Butler, K., Dupuis, J. \& Kepic, A., 2007. Signal to noise improvements in seismoelectrics data acquisition, Ground Borehole Geophys. Methods, 111, 3753-3755.

Butler, K.E. \& Russell, R.D., 2003. Cancellation of multiple harmonic noise series in geophysical records, Geophysics, 68, 1083-1090.

Carcione, J., 2014. Wave Fields in Real Media, 3rd edn, Elsevier Science.

Carcione, J., Seriani, G. \& Gei, D., 2003. Acoustic and electromagnetic properties of soil saturated with salt water and NAPL, J. Appl. Geophys., 52, 177-191.

Carsel, R. \& Parrish, R., 1998. Developing joint probability distributions of soil water characteristics, Water Resour. Res., 24, 755-769.

Chotiros, N. \& Isakson, M., 2004. A broadband model of sandy ocean sediments: Biot-Stoll with contact squirt flow and shear drag, J. acoust. Soc. Am., 116(4), 2011-2022.

Crane, J., Lorenzo, J. \& Harris, J., 2013. A new electrical and mechanically detonatable shear wave source for near surface $(0-30 \mathrm{~m})$ seismic acquisition, J. Appl. Geophys., 91, 1-8.

Darnet, M. \& Marquis, G., 2004. Modelling streaming potential (SP) signals induced by water movement in the vadose zone, J. Hydrol., 285, 114-124.

Darnet, M., Marquis, G. \& Sailhac, P., 2003. Estimating aquifer hydraulic properties from the inversion of surface streaming potential (SP) anomalies, Geophys. Res. Lett., 30(13), doi:10.1029/2003GL017631. 
Dean, T., Dupuis, C., Herrmann, R. \& Valuri, J., 2012. A brute-strength approach to improving the quality of seismoelectric data, in SEG Annual meeting Las Vegas, pp. 1-4, doi:10.1190/segam2012-0222.1.

Dupuis, J.C., Butler, K.E. \& Kepic, A.W., 2007. Seismoelectric imaging of the vadose zone of a sand aquifer, Geophysics, 72, A81-A85.

Dupuis, J.C., Butler, K.E., Kepic, A.W. \& Harris, B.D., 2009. Anatomy of a seismoelectric conversion: measurements and conceptual modeling in boreholes penetrating a sandy aquifer, J. geophys. Res., 114(B13), B10306, doi:10.1029/2008JB005939.

Dupuy, B., Garambois, S. \& Virieux, J., 2016. Estimation of rock physics properties from seismic attributes-Part 1: Strategy and sensitivity analysis, Geophysics, 80(3), M35-M53.

Frenkel, J., 1944. On the theory of seismic and electroseismic phenomena in a moist soil, J. Phys., 8(4), 230-241.

Garambois, S. \& Dietrich, M., 2001. Seismoelectric wave conversions in porous media: field measurements and transfer function analysis, Geophysics, 66, 1417-1430.

Garambois, S., Senechal, P. \& Perroud, H., 2002. On the use of combined geophysical methods to access water content and water conductivity of near surface formations, J. Hydrol., 259, 32-48.

Gauzellino, P., Carcione, J., Santos, J. \& Picotti, S., 2014. A rheological equation for an isotropic-anelastic media and simulation of field seismograms, Wave Motion, 51, 743-757.

Giampaolo, V., Rizzo, E., Titov, K., Konosavsky, P., Laletina, D., Maineult, A. \& Lapenna, V., 2014. Self-potential monitoring of a crude oilcontaminated site (Trecate, Italy), Environ. Sci. Pollut. Res., 21, 89328947.

Girard, J., Legchenko, A. \& Boucher, M., 2005. Stability of MRS signal and estimating data quality, Near Surf. Geophys., 3(3), 187-194.

Guichet, X., Jouniaux, L. \& Pozzi, J.-P., 2003. Streaming potential of a sand column in partial saturation conditions, J. geophys. Res., 108(B3), 2141, doi:10.1029/2001JB001517.

Haines, S.H. \& Pride, S.R., 2006. Seismoelectric numerical modeling on a grid, Geophysics, 71(6), 57-65.

Hayley, K., Bentley, L.R., Gharibi, M. \& Nightingale, M., 2007. Low temperature dependence of electrical resistivity: implications for near surface geophysical monitoring, Geophys. Res. Lett., 34, doi:10.1029/2007GL031124.

Henry, P., Jouniaux, L., Screaton, E.J., S. Hunze \& Saffer, D.M., 2003. Anisotropy of electrical conductivity record of initial strain at the toe of the Nankai accretionary wedge, J. geophys. Res., 108, 2407, doi:10.1029/2002JB002287.

Hu, H. \& Liu, J., 2002. Simulation of the converted electric field during acoustoelectric logging, in 72nd SEG Annual International Meeting, Expanded Abstracts, 21 (Salt Lake City, Utah, USA), pp. 348-351.

Hu, H., Guan, W. \& Harris, J., 2007. Theoretical simulation of electroacoustic borehole logging in a fluid-saturated porous formation, J. acoust. Soc. Am., 122, 135-145.

Ivanov, A., 1939. Effect of electrization of earth layers by elastic waves passing through them (in Russian), Dokl. Akad. Nauk SSSR, 24, 42-45.

Jackson, M.D., 2010. Multiphase electrokinetic coupling: insights into the impact of fluid and charge distribution at the pore scale from a bundle of capillary tubes model, J. geophys. Res., 115, B07206, doi:10.1029/2009JB007092.

Jardani, A., Revil, A., Slob, E. \& Söllner, W., 2010. Stochastic joint inversion of 2D seismic and seismoelectric signals in linear poroelastic materials: A numerical investigation, Geophysics, 75(1), N19-N31.

Jougnot, D., Linde, N., Haarder, E. \& Looms, M., 2015. Monitoring of saline tracer movement with vertically distributed self-potential measurements at the HOBE agricultural site, Voulund, Denmark, J. Hydrol., 521, 314327.

Jouniaux, L. \& Ishido, T., 2012. Electrokinetics in Earth sciences: a tutorial, Int. J. Geophys., 2012, doi:10.1155/2012/286107.

Jouniaux, L. \& Zyserman, F., 2015. Interactive comment on a review on electrokinetically induced seismo-electrics, electro-seismics, and seismo-magnetics for Earth sciences, Author reply to comment of A. Revil., Solid Earth, 7, C1274-C1274, http://www. solid-earth-discuss.net/7/C1274/2015/sed-7-C1274-2015.pdf.
Jouniaux, L. \& Zyserman, F., 2016. A review on electrokinetically induced seismo-electrics, electro-seismics, and seismo-magnetics for Earth sciences, Solid Earth, 7, 249-284.

Jouniaux, L., Lallemant, S. \& Pozzi, J., 1994. Changes in the permeability, streaming potential and resistivity of a claystone from the Nankai prism under stress, Geophys. Res. Lett., 21, 149-152.

Jouniaux, L., Pozzi, J.-P., Berthier, J. \& Massé, P., 1999. Detection of fluid flow variations at the Nankai trough by electric and magnetic measurements in boreholes or at the seafloor, J. geophys. Res., 104, $29293-$ 29309.

Jouniaux, L., Zamora, M. \& Reuschlé, T., 2006. Electrical conductivity evolution of non-saturated carbonate rocks during deformation up to failure, Geophys. J. Int., 167, 1017-1026.

Krawczyk, C., Polom, U., Trabs, S. \& Dahm, T., 2012. Sinkholes in the city of Hamburg - new urban shear-wave reflection seismic system enables high-resolution imaging of subrosion structures, J. Appl. Geophys., 78, 133-143.

Krawczyk, C., Polom, U. \& Beilecke, T., 2013. Shear-wave reflection seismics as a valuable tool for near-surface urban applications, Leading Edge, 32(3), 253-263.

Lerwill, W.E., 1981. The amplitude and phase response of a seismic vibrator, Geophys. Prospect., 29, 503-528.

Liu, X. \& Greenhalgh, S., 2014. Reflection and transmission coefficients for an incident plane shear wave at an interface separating two dissimilar poroelastic solids, Pure appl. Geophys., 171, 2111-2127.

Liu, H.-P., Don, L. \& Kanamori, H., 1976. Velocity dispersion due to anelasticity: implications for seismology and mantle composition, J. geophys. Res., 47(1), 41-58.

Long, L.T. \& Rivers, W.K., 1975. Field measurement of the electroseismic response, Geophysics, 40, 233-245.

Maineult, A., Jouniaux, L. \& Bernabé, Y., 2006. Influence of the mineralogical composition on the self-potential response to advection of $\mathrm{kcl}$ concentration fronts through sand, Geophys. Res. Lett., 33, L24311, doi:10.1029/2006GL028048.

Martner, S.T. \& Sparks, N.R., 1959. The electroseismic effect, Geophysics, 24(2), 297-308.

Mavko, G., Mukerji, T. \& Dvorkin, J., 2009. The Rock Physics Handbook: Tools for Seismic Analysis of Porous Media, Cambridge Univ. Press.

Mboh, C.M., Huisman, J.A., Zimmermann, E. \& Vereecken, H., 2012. Coupled hydrogeophysical inversion of streaming potential signals for unsaturated soil hydraulic properties, Vadose Zone J., 11(2), doi:10.2136/vzj2011.0115.

Munch, F. \& Zyserman, F., 2016. Detection of non-aqueous phase liquids contamination by SH-TE seismoelectrics: a computational feasibility study, J. Appl. Geophys., 130, 8-22.

Perrier, F. \& Morat, P., 2000. Characterization of electrical daily variations induced by capillary flow in the non-saturated zone, Pure appl. Geophys., 157, 785-810.

Pinettes, P., Bernard, P., Cornet, F., Hovhannissian, G., Jouniaux, L., Pozzi, J.-P. \& Barthés, V., 2002. On the difficulty of detecting streaming potentials generated at depth, Pure appl. Geophys., 159, 26292657.

Pride, S.R., 2005. Relationships between seismic and hydrological properties, in Hydrogeophysics, chap. 9, pp. 253-291, Springer.

Pride, S., 1994. Governing equations for the coupled electromagnetics and acoustics of porous media, Phys. Rev. B, 50, 15 678-15 695.

Pride, S. \& Haartsen, M.W., 1996. Electroseismic wave properties, J. acoust. Soc. Am., 100, 1301-1315.

Pride, S. \& Morgan, F.D., 1991. Electrokinetic dissipation induced by seismic waves, Geophysics, 56(7), 914-925.

Revil, A. \& Linde, N., 2006. Chemico-electromechanical coupling in microporous media, J. Colloid Interface Sci., 302, 682-694.

Revil, A. \& Mahardika, H., 2013. Coupled hydromechanical and electromagnetic disturbances in unsaturated porous materials, Water Resour. Res., 49, 744-766.

Revil, A., Linde, N., Cerepi, A., Jougnot, D., Matthäi, S. \& Finsterle, S., 2007. Electrokinetic coupling in unsaturated porous media, J. Colloid Interface Sci., 313, 315-327. 
Revil, A., Barnier, G., Karaoulis, M., Sava, P., Jardani, A. \& Kulessa, B., 2014. Seismoelectric coupling in unsaturated porous media: theory, petrophysics, and saturation front localization using an electroacoustic approach, Geophys. J. Int., 196(2), 867-884.

Revil, A., Jardani, A., Sava, P. \& Haas, A., 2015. The Seismoelectric Method: Theory and Application, Wiley Blackwell.

Richards, L., 1931. Capillary conduction of liquids through porous mediums, Physics, 1, 318-333.

Russell, R.D., Butler, K.E., Kepic, A.W. \& Maxwell, M., 1997. Seismoelectric exploration, Leading Edge, 16(11), 1611-1615.

Sailhac, P., Darnet, M. \& Marquis, G., 2004. Electrical streaming potential measured at the ground surface: forward modeling and inversion issues for monitoring infiltration and characterizing the vadose zone, Vadose Zone J., 3, 1200-1206.

Santos, J., Ravazzoli, C., Gauzellino, P., Carcione, J. \& Cavallini, F., 2004. Simulation of waves in poro-viscoelastic rocks saturated by immiscible fluids. numerical evidence of a second slow wave, J. Comput. Acoust., 12, $1-21$.

Santos, J., Ravazzoli, C., Gauzellino, P. \& Carcione, J., 2005. Numerical simulation of ultrasonic waves in reservoir rocks with patchy saturation and fractal petrophysicas properties, Comput. Geosci., 9, 1-27.

Saunders, J.H., Jackson, M.D. \& Pain, C.C., 2008. Fluid flow monitoring in oilfields using downhole measurements of electrokinetic potential, Geophysics, 73, E165-E180.

Schön, J., 1996. Handbook of Geophysical Exploration, Seismic Exploration, vol. 18, Elsevier Science Ltd.

Smeulders, D.M.J., Grobbe, N., Heller, H.K.J. \& Schakel, M., 2014. Seismoelectric conversion for the detection of porous medium interfaces between wetting and nonwetting fluids, Vadose Zone J., 13, 1-7.

Strahser, M., Jouniaux, L., Sailhac, P., Matthey, P.-D. \& Zillmer, M., 2011. Dependence of seismoelectric amplitudes on water-content, Geophys. $J$. Int., 187, 1378-1392.
Teja, A.S. \& Rice, P., 1981. Generalized corresponding states method for the viscosities of liquid mixtures, Ind. Eng. Chem. Fundam., 20(1), 77-81.

Thompson, A., Sumner, J. \& Hornbostel, S., 2007. Electromagnetic-toseismic conversion: a new direct hydrocarbon indicator, Leading Edge, 26, 428-435.

Turesson, A., 2006. Water content and porosity estimated from ground-penetrating radar and resistivity, J. Appl. Geophys., 58, 99-111.

U.S. Soil Conservation Service, 1987. Soil Mechanics Level 1, Module 3, USDA Textural Classification Study Guide, United States Department of Agriculture.

Valuri, J., Dean, T. \& Dupuis, J., 2012. Seismoelectric acquisition in an arid environment, in 22nd International Geophysical Conference and Exhibition, Brisbane, Australia.

van Genuchten, M., 1980. A closed form equation for predicting the hydraulic conductivity of unsaturated soils, Soil. Sci. Soc. Am. J., 40, 892 898.

Warden, S., Garambois, S., Sailhac, P., Jouniaux, L. \& Bano, M., 2012. Curvelet-based seismoelectric data processing, Geophys. J. Int., 190, $1533-1550$

Warden, S., Garambois, S., Jouniaux, L., Brito, D., Sailhac, P. \& Bordes, C., 2013. Seismoelectric wave propagation numerical modeling in partially saturated materials, Geophys. J. Int., 194, 1498-1513.

Zyserman, F., Gauzellino, P. \& Santos, J., 2010. Finite element modeling of SHTE and PSVTM electroseismics, J. Appl. Geophys., 72, 79-91.

Zyserman, F., Gauzellino, P. \& Santos, J., 2012. Numerical evidence of gas hydrate detection by means of electroseismics, J. Appl. Geophys., 86, 98-108.

Zyserman, F., Jouniaux, L., Warden, S. \& Garambois, S., 2015. Borehole seismoelectric logging using a shear-wave source: possible application to $\mathrm{CO}_{2}$ disposal?, Int. J. Greenhouse Gas Control, 33, 82-102. 